\title{
Formation of Specific Afferent Connections in Organotypic Slice Cultures from Rat Visual Cortex Cocultured with Lateral Geniculate Nucleus
}

\author{
Jürgen Bolz, ${ }^{1}$ Nino Novak, ${ }^{1}$ and Volker Staiger ${ }^{2}$ \\ 'Friedrich-Miescher-Labor der Max-Planck-Gesellschaft and ${ }^{2}$ Max-Planck Institut für biologische Kybernetik, 7400 \\ Tübingen, Germany
}

The development of the cerebral cortex involves the specification of intrinsic circuitry and extrinsic connections, the pattern of inputs and outputs. To investigate the development of a major afferent input to the cortex, we studied the formation of thalamocortical connections in an organotypic culture system. Slices from the lateral thalamus of young rats were cocultured with slices from the visual cortex. Thalamocortical projections in vitro were examined anatomically with fluorescent dyes and physiologically with electrophysiological and optical recording techniques.

Axons emerged from thalamic explants radially in all directions. The outgrowth of thalamic fibers and the course of the axonal trajectories were not influenced by the presence of the cocultured cortex. Only those thalamic axons that happened to grow toward the cortical slices invaded their target tissue. Thalamocortical projection cell in vitro had the characteristic morphology of thalamic relay neurons. Cells with the morphology of interneurons were present in thalamic explants, but these neurons did not project to the cocultured cortex. Thalamocortical axons in vitro terminated in their appropriate cortical target layer, formed axonal arbors, and made functional synaptic contacts. Such specific connections between thalamic neurons and their cortical target cells were established regardless of whether thalamocortical axons invaded the cortex from the white matter side or from the pial surface. These results suggest that thalamic projection neurons have an innate mechanism that allows them to recognize their cortical target cells. Thus, intrinsic factors play a significant role in the laminar specification of cortical connections during development.

The neuronal connections between the thalamus and the cerebral cortex are organized with great precision. Each sensury nucleus of the thalamus projects to a specific area of the neocortex and, in turn, receives input from the area of cortex to which it projects. Thalamic relay neurons form an ordered topographical projection within their cortical target region where they contact primarily neurons in layer 4 , while cells in layer 6 project back to their thalamic nucleus. The generation of these

Received Nov. 4, 1991; revised Feb. 25, 1992; accepted Mar. 11, 1992.

We are grateful to Magdalena Götz for help in some of the experiments and many stimulating discussions. We thank Renate Thanos and Iris Kehrer for superb technical assistance, and Mary Behan for helpful comments on the manuscript.

Correspondence should be addressed to Jürgen Bolz, Friedrich-Miescher-Labor der Max-Planck-Gesellschaft, Spemannstrasse 37-39, 7400 Tübingen, Germany. Copyright (C) 1992 Society for Neuroscience $0270-6474 / 92 / 123054-17 \$ 05.00 / 0$ specific reciprocal connections between thalamus and cortex involves a complex sequence of developmental events that have been studied most thoroughly in the visual system. For example, previous studies have established that axons from the LGN arrive at the developing visual cortex before their target cells in layer 4 are generated in the proliferative region of the ventricular surface (Lund and Mustari, 1977; Rakic, 1977; Shatz and Luskin, 1986). However, the thalamocortical axons do not invade the cortex but first grow into the subplate zone, a region situated bclow the cortical plate that contains the carlicst-gencrated ncurons (Kostovic and Rakic, 1980; Luskin and Shatz, 1985; Chun et al., 1987; Wahle et al., 1987; Chun and Shatz, 1989a,b; Wahle and Mayer, 1989). Thalamic afferents innervate the cortex only after layer 4 cells have been generated and have migrated from the ventricular zone toward their appropriate position in the cortical plate.

The above-mentioned observations have led to a number of suggestions as to the mechanisms by which specific interconnections between thalamus and cortex are achieved during development. For example, because thalamic fibers "wait" in the subplate zone before they enter the developing cortex, it has been proposed that the state of maturation of the cortex determines the ingrowth of cortical afferents (Wise and Jones, 1976, 1978). It has also been hypothesized that the subplate serves as a temporary target to promote the waiting of afferents (Rakic, 1977; Kostovic and Rakic, 1980, 1990; Luskin and Shatz, 1985; Chun and Shatz, 1989a,b). However, based on observations made in the intact animal, these suggestions are for the most part supported only by correlative evidence.

An in vitro system is of considerable advantage in gaining some insight into the cellular and molecular strategies by which connections between thalamus and cortex are established. Various methods have been described to maintain the mammalian cortex in culture (Crain and Bornstein, 1964; Seil et al., 1974; Romijn et al., 1988; Caeser et al., 1989; Wolfson et al., 1989; Annis et al., 1990). In previous studies, a roller culture method (Gähwiler, 1981a) was used to cultivate slices from the visual cortex of young postnatal rats for several months. It has been demonstrated that such cortical slice cultures are organotypically organized and that cortical neurons, at least to some extent, continue to differentiate and mature in these preparations (Caeser et al., 1989; Götz and Bolz, 1989, 1990; Annis et al., 1990; Bolz et al., 1990; Wolburg and Bolz, 1991). Cells in cortical cultures establish connections with appropriate cocultured brain areas (Yamamoto et al., 1989; Bolz et al., 1990; Heffner et al., 1990; Molnar and Blakemore, 1991). These findings have opened 
up the possibility of studying aspects of development and function of cortical connections under well-controlled experimental conditions. In the present study, we examine the afferent projection from thalamic slices to cocultured slices from visual cortex and show that these connections are functional and terminate in the appropriate cortical layer.

\section{Materials and Methods}

Preparation of slice cultures. Cortical slice cultures were prepared using a roller culture technique (Gähwiler, 1981a) as described previously by Caeser et al. (1989). In brief, rat pups (Lewis) from postnatal day 0 (P0) up to $P 9$ were decapitated, and small blocks of the dorsal occipital cortex from both hemispheres were prepared aseptically in ice-cold Gey's balanced salt solution (GBSS) with $6.5 \mathrm{mg} / \mathrm{ml}$ glucose. The pia was taken off and tissue blocks were cut coronally with a McIlwain tissue chopper at a thickness of $300 \mu \mathrm{m}$. For preparation of thalamic slices the cortical hemispheres were first removed and the diencephalon was then incised vertically, anterior and posterior to the LGN. This provided a tissue block of about $1 \mathrm{~mm}$ thickness whose dorsolateral portions contained the LGN of both hemispheres. The LGN were separated from this block by two oblique incisions running from dorsomedial to ventrolateral. The remaining pia was carefully removed and the blocks were sliced at $300 \mu \mathrm{m}$.

After cutting, the slices were stored for $30 \mathrm{~min}$ at $4^{\circ} \mathrm{C}$ in GBSS. Subsequently, a thalamic slice was placed next to a cortical slice (distance about $0.5-1 \mathrm{~mm}$ ) on a cleaned coverslip and embedded in a plasma clot of $20 \mu \mathrm{l}$ of citrated chicken plasma (Sigma) coagulated by $20 \mu \mathrm{l}$ of thrombin solution $(0.2 \mathrm{mg} / \mathrm{ml}, 20 \mathrm{NIH}$-units $/ \mathrm{ml}$, Hoffmann La Roche). The thalamic slice was placed either next to the white matter or next to the pial side of the cortical slice. In some experiments, a thalamic slice was cocultured with a cortical slice prepared from animals of the same age, and in others, with a cortical slice prepared from animals of different ages.

The cocultures were maintained in plastic culture tubes (Nunc) with $0.75 \mathrm{ml}$ of medium consisting of $50 \%$ Eagle's basal medium, $25 \%$ Hanks' balanced salt solution, and $25 \%$ horse serum containing $0.1 \mathrm{~mm}$ glutamine and $6.5 \mathrm{mg} / \mathrm{ml}$ glucose (all components from GIBCO). The medium was changed every other day. The culture tubes were put in a roller drum incubator at $36^{\circ} \mathrm{C}$ (dry air without $\mathrm{CO}_{2} / \mathrm{O}_{2}$ control) that rotated at 14 revolutions per hour. After $3 \mathrm{~d}$ in culture, mitotic inhibitors (5-fluoro-2-deoxyuridine, cytosine- $\beta$-D-arabinofuranoside, uridine; all from Sigma) were added for $24 \mathrm{hr}$ at a final concentration of $4.4 \mu \mathrm{M}$ to prevent excessive growth of glia cells and fibroblasts.

In some experiments, single cortical slices were first kept in vitro for 3-9 d, and then cocultured with freshly prepared thalamic slices. Cocultures obtained with this technique received antimitotics only once, before the thalamus was added.

Electrophysiological recording. Electrophysiological recordings were carried out in a temperature-controlled chamber at $32^{\circ} \mathrm{C}$. The chamber was mounted on an inverted microscope (Zeiss IM 35). The cultures were constantly superfused with $30 \mathrm{ml} / \mathrm{hr}$ Hanks' balanced salt solution (GIBCO) with additional $2 \mathrm{~mm} \mathrm{CaCl}$. For intracellular recordings, we used glass electrodes pulled from thick-wall glass capillaries (Clark) filled with $3 \mathrm{M} \mathrm{K}$-acetate or $3 \mathrm{M} \mathrm{KCl}$. The electrodes had resistances between 60 and $90 \mathrm{M} \Omega$.

Thalamic cultures were electrically stimulated with single current pulses applied to monopolar insulated tungsten electrodes (duration, $50 \mu \mathrm{sec}$; amplitude, $10-1000 \mu \mathrm{A}$; electrode negative). For pharmacological stimulation, glutamate ( $3 \mathrm{M}, \mathrm{pH} 8.4$ ) was applied iontophoretically through glass electrodes with resistances of approximately $10 \mathrm{M}$. Glutamate was ejected with currents between 30 and $500 \mathrm{nA}$. Recordings were documented either by taking photographs from the screen of a storage oscilloscope or by storing digitized data in a computer.

Optical recording. Voltage-sensitive dyes were used to monitor electrical activity over a large area in cortical explants in response to electrical stimulation of the cocultured thalamic slice. In these experiments, we used the styryl dyes RH237 or RH795 (Grinvald et al., 1982, 1984). The medium in the chamber was exchanged for a medium to which the dye was added at about a $1 \mu \mathrm{M}$ concentration, and the perfusion was then stopped for 15-30 min to allow for staining with the dye. After the perfusion recommenced, we waited about $20 \mathrm{~min}$ to ensure that any dye not bound to membranes was washed out. The cocultures were illuminated with light from a mercury lamp with stabilized power supply
(Siemens) passed through an epifluorescence filter set (Zeiss, BP 546, FT 580, LP 590). The image of the slice culture was projected with an objective (Leitz $10 \times$, NA 0.45 or Zeiss $63 \times$, NA 1.25 , oil immersion) onto a $12 \times 12$ photodiode array (Centronics, M144-5), which transformed the fluorescent signals into electrical signals. These signals were amplified, digitized, and fed into a computer for further processing as described previously (Bonhoeffer and Staiger, 1988).

As with the electrophysiological experiments, we stimulated the thalamic slice with monopolar tungsten electrodes and simultaneously recorded the fluorescence signals over a period of either 96 or $192 \mathrm{msec}$. To minimize photodynamic damage, a relatively long interstimulus interval of $30 \mathrm{sec}$ was used. At each position, we recorded two to four sweeps, and then moved the objective to a different location over the cortical culture. We attempted to record optically from the whole cortical slice, but we chose the recording sites randomly so that a possible drift in the optical signals over the course of an experiment would not be translated into a spatial gradient. Moreover, to control for the stability of the signals, we recorded from several locations repetitively at different times during an experiment. An experiment was stopped once optical signals at a given site began to change.

Histology and tracing. For intracellular staining of individual neurons, cell bodies were impaled with Lucifer yellow-filled electrodes (10-20\% in $0.1 \mathrm{M} \mathrm{LiCl}$; Aldrich). Lucifer yellow was injected into the cells with negative current pulses $(1-4 \mathrm{nA}, 200 \mathrm{msec}$ duration at $4 \mathrm{~Hz})$ applied to the electrode for 3-15 min. After several cells had been injected, the cultures were fixed with $4 \%$ paraformaldehyde in $0.1 \mathrm{~m}$ phosphate buffer, $\mathrm{pH} 7.4(\mathrm{~PB})$ for several hours, dehydrated in an ascending alcohol series, cleared with xylene, and mounted in Entellan (Merck). Lucifer yellowfilled cells were examined with a Leitz epifluorescence microscope (Leitz I2.3 filter set). Selected cells were photographed and drawn with the aid of a drawing apparatus.

For retrograde and anterograde tracing, we used the lipophilic fluorescent dye dioctadecyl-tetramethylindocarbocyanine perchlorate (DiI; Molecular Probes, Eugene, OR). Several very small Dil crystals were placed in either the thalamic or the cortical explants. The cocultures were then fixed with 4\% paraformaldehyde in PB and kept in the fixative at room temperature for 1-3 months to allow diffusion of the dye (Godement et al., 1987). The labeled cells and fibers were viewed with a Leitz epifluorescence microscope equipped with a Leitz N 2.1 (rhodamine) filter set and either photographed or reconstructed using a drawing apparatus.

To examine the histological appearance of the thalamic explants and to study the layering of the cortical explants, most cocultures were counterstained with $1 \%$ cresyl violet in distilled water, differentiated in acetic acid-alcohol, dehydrated in ascending alcohols, cleared in xylene, and embedded in Entellan (Merck). In some experiments, cells destined for different layers were labeled on their birthday by injecting timed pregnant rats with $250 \mathrm{mg} / \mathrm{kg}$ body weight 5 -bromodeoxyuridine ( $\mathrm{BrdU}$ ); the day of sperm detection was defined as embryonic day 1 (El). Cells that are generated at the time of injection incorporate BrdU. They were detected in slice cultures and in littermates of the corresponding age with immunohistochemical techniques (Miller and Nowakowski, 1988). BrdU-immunoreactive cells were drawn from the microscope with a drawing apparatus; from these reconstructions, histograms were made representing the distribution of labeled cells at different relative depths in the cortex.

\section{Results}

\section{Cellular organization of cortical and thalamic slice cultures}

Several previous studies described the structural organization of cortical slice cultures at both the light and electron microscopic level (Caeser et al., 1989; Götz and Bolz, 1989, 1990; Annis et al., 1990; Bolz et al., 1990; Wolburg and Bolz, 1991); thus, only a few of the relevant features are noted here. Cortical slice cultures prepared from rat pups on P0 up to P11 can be maintained in vitro for several months. At the time of preparation of the cultures, cortical slices are cut at a thickness of 300 $\mu \mathrm{m}$, but after $1-2$ weeks in vitro they flatten to about $40-100$ $\mu \mathrm{m}$. Cortical slice cultures contain pyramidal cells and different classes of nonpyramidal cells with the typical morphological and neurochemical features of their in vivo counterparts. U1trastructurally, the characteristic pattern of symmetric and 


\section{BrdU birthdating}

\section{in vivo}

Figure 1. Layering of cortical slice cultures determined by birthdating with BrdU. This composite drawing shows the distribution of BrdU-immunoreactive cells in vivo (left) and in vitro (right) after BrdU injections on E16 (triangles), E17 (open circles), and E18 (solid circles). The numbers on the left indicate the cortical layers in vivo; the broken lines represent the borders between the gray and white matter. Slice cultures were prepared from 0-2-d-old animals and kept in vitro for 2-3 weeks. BrdUlabeled cells in vivo were examined in littermates of the corresponding age. A quantitative analysis of the results from a BrdU injection on E17 is illustrated in Figure 7.

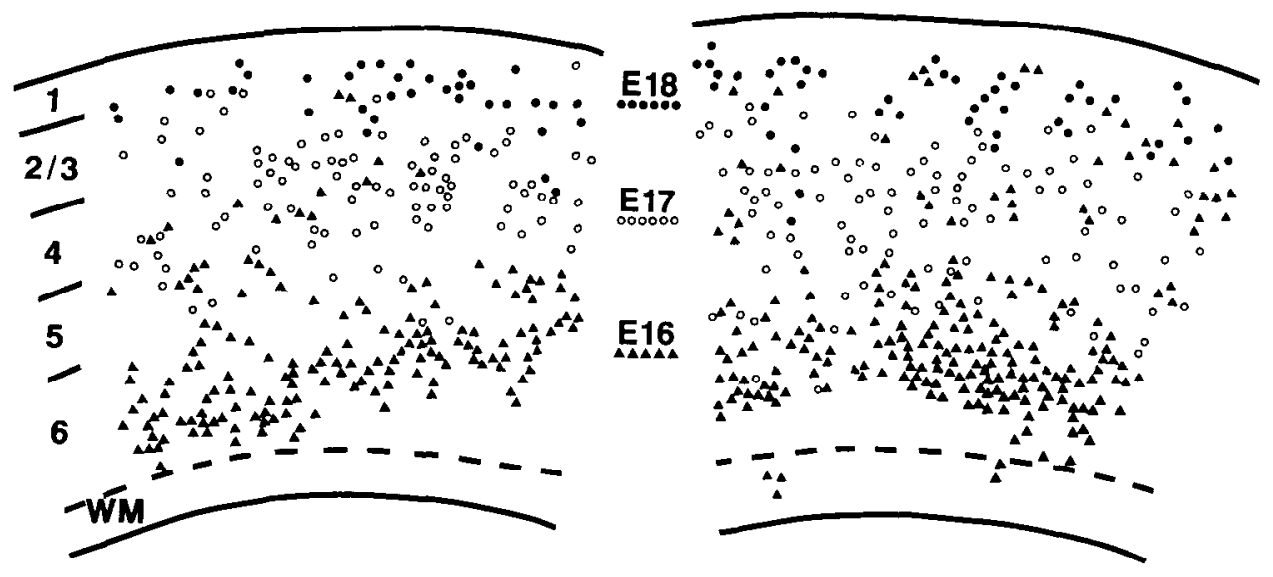

asymmetric synaptic connections in slice cultures is similar to that observed in normal cortex. Nissl staining revealed that the cytoarchitecture is preserved in slice cultures. There is almost always a sharp border delineating the gray matter from the underlying white matter zone, and the cortical layers are recognizable. However, it is not always possible to determine the exact boundary of every cortical layer. This is somewhat similar to the situation in situ, where the cortical layers of young rats are less distinct in Nissl-stained sections than in adult animals.

The layering of cortical slice cultures was also assessed with a different method. It is well known that the cells destined for the various cortical layers are born at about the same time. The generation of cortical neurons follows an inside-first, outsidelast sequence, with the cells in the later-generated upper layers migrating past those in the earlier-generated lower layers (Angevine and Sidman, 1961; Berry and Rogers, 1965; Hicks and D'Amato, 1968; Rakic, 1974; Luskin and Shatz, 1985). As described in more detail elsewhere (Götz and Bolz, 1992), cells were labeled on their birthday by injecting timed pregnant rats with BrdU, and the location of BrdU-labeled cells in slice cultures after 1-3 weeks in vitro was compared with the distribution of labeled cells in littermates of the corresponding age. As reported previously, $87 \%$ of the cells generated on E16 are located in layers 5 and 6 in vivo and $71 \%$ of the BrdU-labeled cells are found in the lower half of the gray matter in slice cultures. With an injection of BrdU on E18, 93\% of the labeled cells are in layers 2 and 3 in vivo and $89 \%$ of the labeled cells are in the upper quarter of the gray matter in slice cultures (Bolz et al., 1990; Götz and Bolz, 1992). For the present study, we wished to determine the distribution of cells destined for layer 4 and therefore made BrdU injections at E17. In rat cortex, at E17 there is the peak of neurogenesis, and some cells of every cortical layer are born at this embryonic stage (Miller, 1988). However, $53 \%$ of the cells labeled at E17 were located in layer 4, and in slice cultures there was a band of labeled cells at a similar position as in vivo. This is illustrated in Figure 1, which shows the position of cells born at E16, F17, and E18 in vivo and in cortical slice cultures. For a quantitative analysis, we divided the cortical gray matter in 10 equidistant bins and counted the number of cells labeled with BrdU at E17. In vivo, $72 \%$ of the labeled cells were located in bins $6-8$ (bin 1 is adjacent to the white matter side; bin 10 is adjacent to the pial side), and in cortical cultures
$59 \%$ of the labeled cells were found at the same radial position (see Fig. $7 D, E$ ). As will be described in detail below, this is the zone where most afferents from cocultured thalamic explants terminate, and we refer to this region as "the middle" of the cortical slice culture.

Thalamic slices, in contrast to cortical slices, proved to be more difficult to maintain in culture. Thalamic cultures thinned out faster and more irregularly than cortical slice cultures. The inhomogeneous flattening of thalamic slice cultures often resulted in clusters of cell sparse regions. After about 1 week in vitro, the cells in these regions became necrotic and, as a consequence, holes appeared in the plasma clot. Approximately $30 \%$ of thalamic cultures prepared from 0-2-d-old animals and maintained in vitro for $1-4$ weeks flattened homogeneously. These nearly monolayer cultures had clearly defined borders when examined with phase-contrast or dark-field microscopy, indicating that only a few cells had migrated out of the explant (Fig. 2A). Nissl staining showed regularly dispersed neurons in the thalamic slices with small to midsized somata, often triangular in shape (Fig. $2 B$ ). The success rate was only about $20 \%$ for thalamic slice cultures prepared from 3-5-d-old animals and less than $10 \%$ when the animals were older than $5 \mathrm{~d}$. In contrast, it was much easier to obtain good cultures from cortical slices, and this was independent of the age of the animals. More than $80 \%$ of cortical slice cultures prepared from 0-9-d-old rats flattened evenly and showed no signs of degeneration.

In an attempt to overcome some of the difficulties encountered with thalamic slice cultures, we first cultured cortical slices for 3-14 $\mathrm{d}$ in isolation, and then added a freshly prepared thalamic slice to the "precultured" cortex. The coculture was grown for an additional 4-14 d in vitro (DIV) until both the cortical slice and the thalamic slice flattened to nearly monolayers. However, this culture technique did not improve the success rate substantially. Because of these problems with thalamic explants, we had to prepare over 2000 thalamus-cortex cocultures. For the anatomical and physiological experiments described in the present article, only such slice cultures were used that flattened homogeneously, had distinct borders, and showed no obvious indication of degenerating neurons.

To examine the morphology of thalamic neurons in slice cultures, we injected individual neurons intracellularly with Lucifer yellow in living cultures, and labeled cells with the fluorescent 

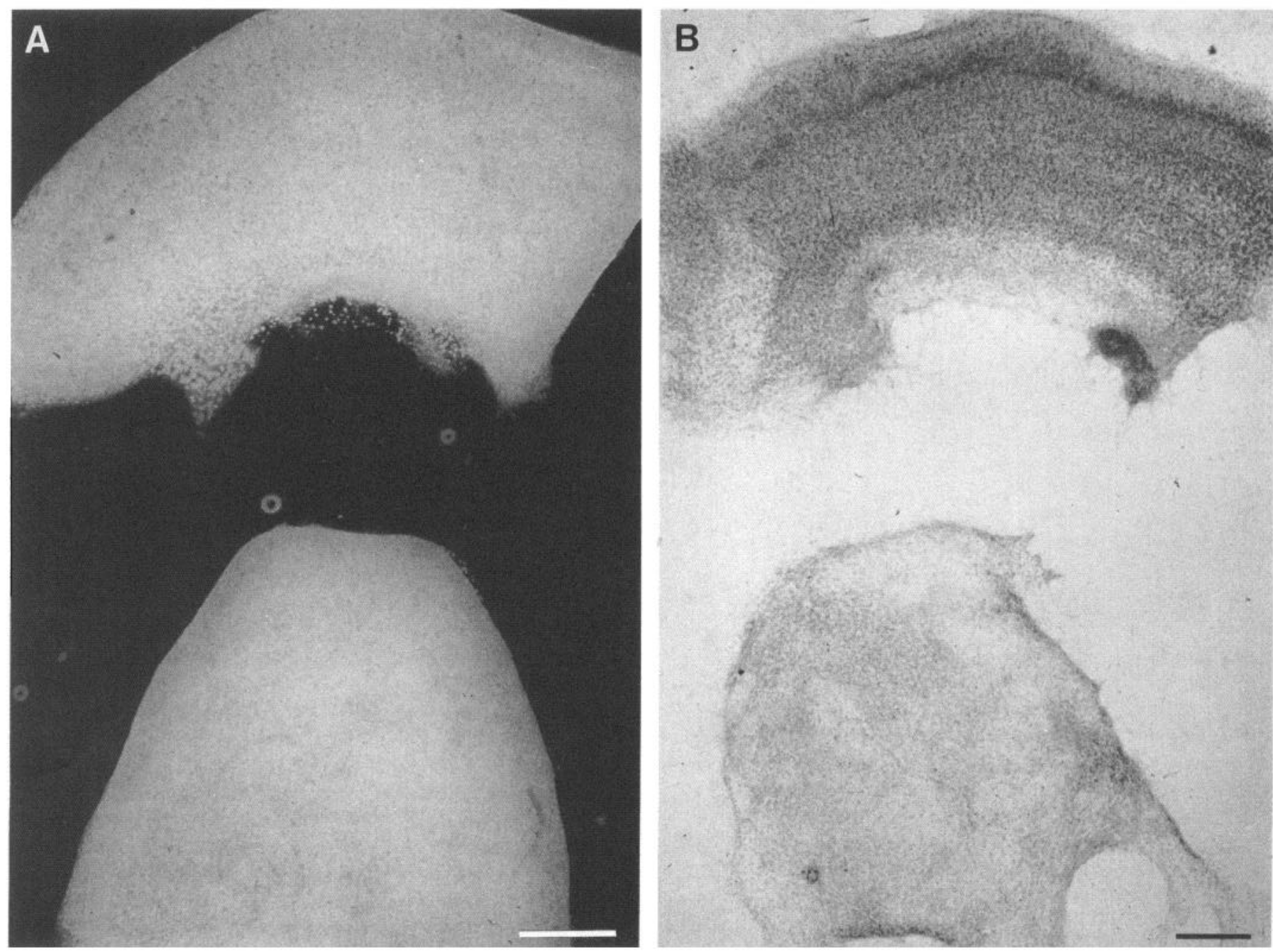

Figure 2. Cocultures of slices from visual cortex (top) and lateral thalamus (bottom) after 12 DIV. The pial side of the cortical slice is up, and the white matter side faces the cocultured thalamus. $A$ is a dark-field micrograph of a live coculture. $B$ illustrates the histological appearance of a fixed coculture stained with cresyl violet (in bright-field optics). Scale bars, $500 \mu \mathrm{m}$.

dye DiI in fixed cultures. Figure 3 shows representative examples of thalamic cells stained in vitro. The cell in Figure $3 A$ has a multipolar morphology with many highly branched primary dendrites that arise from a round soma. In contrast, the cells in Figure $3, B$ and $C$, appear bipolar; they have oval or spindle shaped cell bodies and few primary dendrites that are sparsely branched. Multipolar and bipolar cells in thalamic slice cultures resemble closely class A and class B cells, respectively, of the rat LGN in vivo, first described in Golgi preparations by Grossman et al. (1973). Class A (multipolar) neurons are geniculocortical projection cells, because cells of this class were labeled after HRP injections into the visual cortex, while class B (bipolar) cells were not labeled and therefore are thalamic interneurons (Webster and Rowe, 1984). To determine the morphology of thalamocortical cells in vitro, we labeled cells in thalamic cultures retrogradely with DiI from the cocultured cortical slice. As shown in Figure 4, thalamic projection neurons in vitro are multipolar in shape; cells with bipolar morphology were not found to establish connections with the cocultured cortex.

\section{Thalamocortical connections in vitro: anatomy}

Outgrowing thalamic fibers were examined in living cultures with phase-contrast microscopy, or traced in fixed cultures with DiI and viewed with an epifluorescence microscope. Unstained thalamic fibers growing in the plasma clot were clearly visible with phase-contrast optics, but once the fibers entered the cortex they were only detectable after staining with the fluorescent dye DiI. Crystals of DiI applied to the thalamus produced an intense staining of individual axons and their terminals in the distant cocultured cortex. However, only $10-30 \%$ of all fibers seen with phase-contrast optics were stained with DiI. The unstained axons were probably not in close contact with Dil crystals such that the dye could not diffuse in the membranes of the fibers (Godement et al., 1987). Thus, this technique obviously underestimates the number of outgrowing fibers.

In thalamic slice cultures prepared from 0-2-d-old rats, as early as after 1-2 DIV many fibers could be seen to leave the explant (Fig. $5 A, B$ ). The fiber outgrowth was directed radially away from the thalamic slices, and growth cones were visible at the end of the axons. The axons rarely ramified outside the 

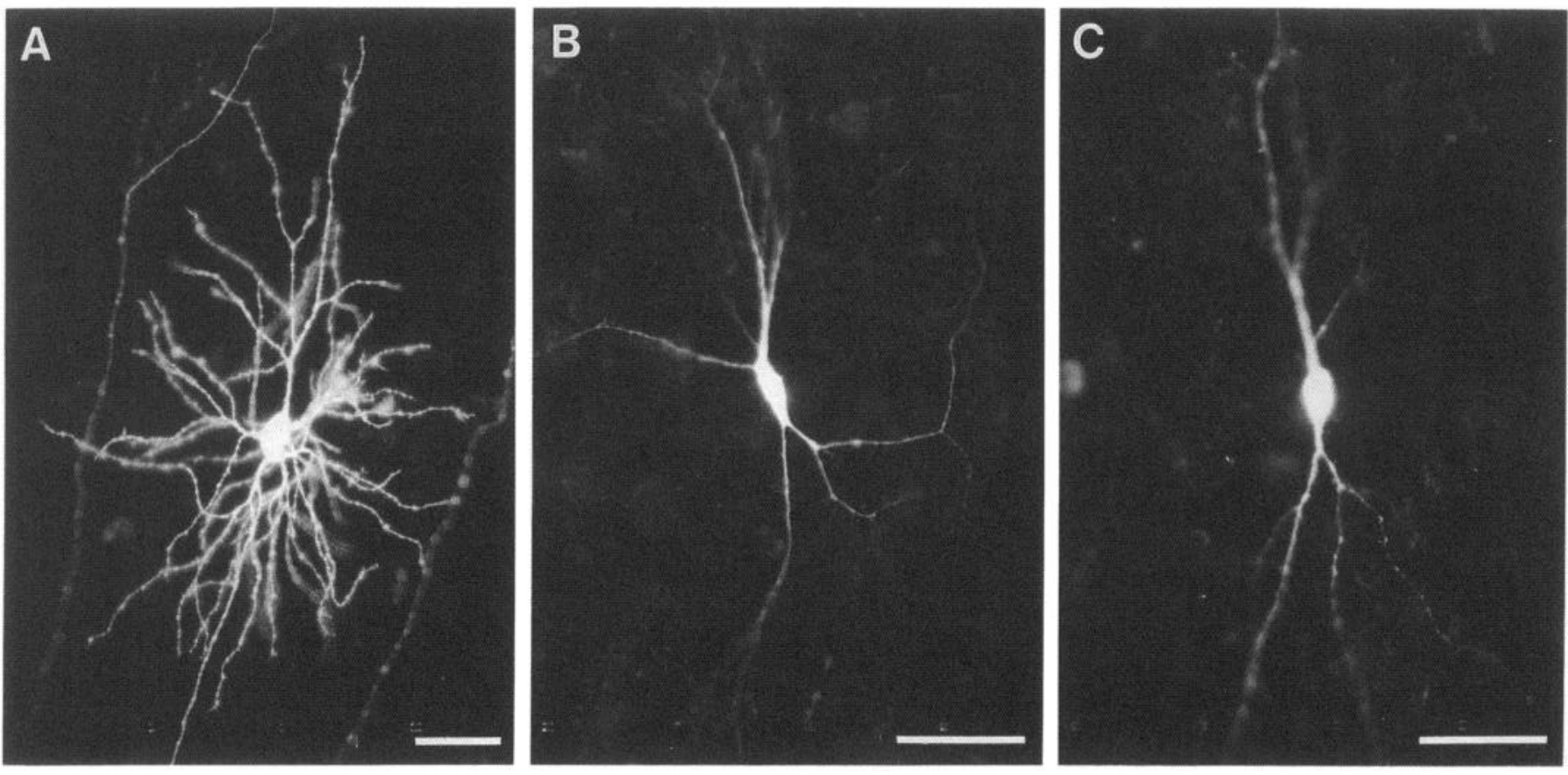

Figure 3. Neurons in thalamic slice cultures injected intracellularly with Lucifer yellow. Slices prepared from P1 rats and cultured for 10-14 d. $A$, Cell with multipolar morphology. $B$ and $C$, Cells with bipolar morphology. Scale bars, $50 \mu \mathrm{m}$.

explant, but when they did, the direction of the fiber branches deviated only slightly from the trajectory of their parent fiber. In some thalamic cultures, fibers left the explant radially in all directions; in others thick fiber bundles were formed at only a few places on the explants. We found no influence of the cocultured cortical target tissue on the fiber outgrowth from the thalamic explants. There was neither an increase in the number of fibers in cortex-thalamus cocultures when compared with thalamic explants cultured alone, nor a directed growth of thalamic fibers toward the cocultured cortex.

The pattern of fiber outgrowth started to change after about 5 DIV, when the fibers reached a length between 2 and $3 \mathrm{~mm}$. Many thalamic fibers that had not reached the cocultured cortex began to degenerate and disappeared after 7-10 DIV. On the
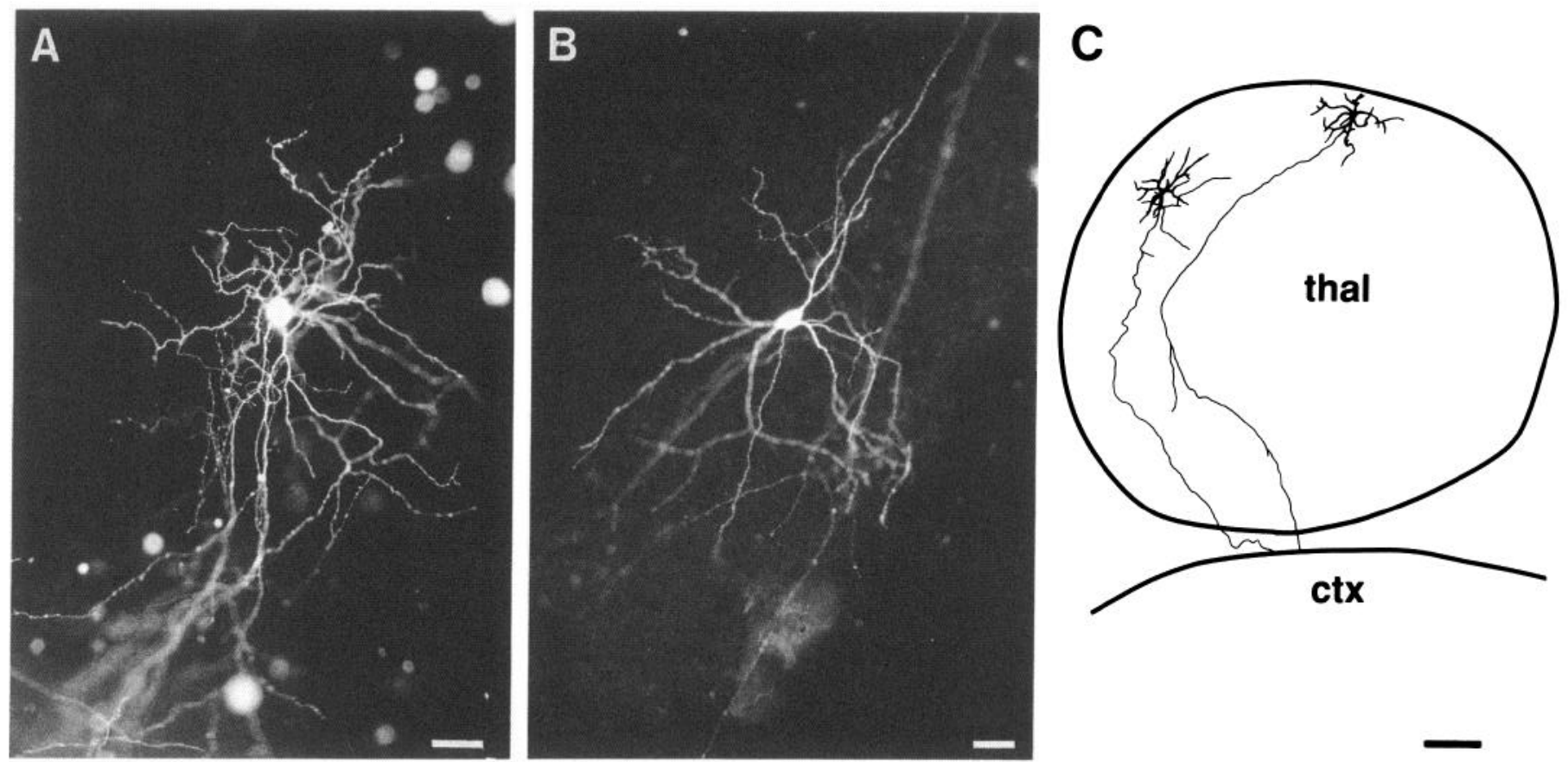

Figure 4. Fluorescent micrographs $(A, B)$ and camera lucida drawings $(C)$ of neurons in thalamic explants backlabeled with Dil from a cocultured slice of visual cortex. Note multipolar morphology of thalamocortical projection neurons in vitro. thal, thalamus; $c t x$, cortex. Scale bars: $A$ and $B$, $50 \mu \mathrm{m} ; C, 500 \mu \mathrm{m}$. 

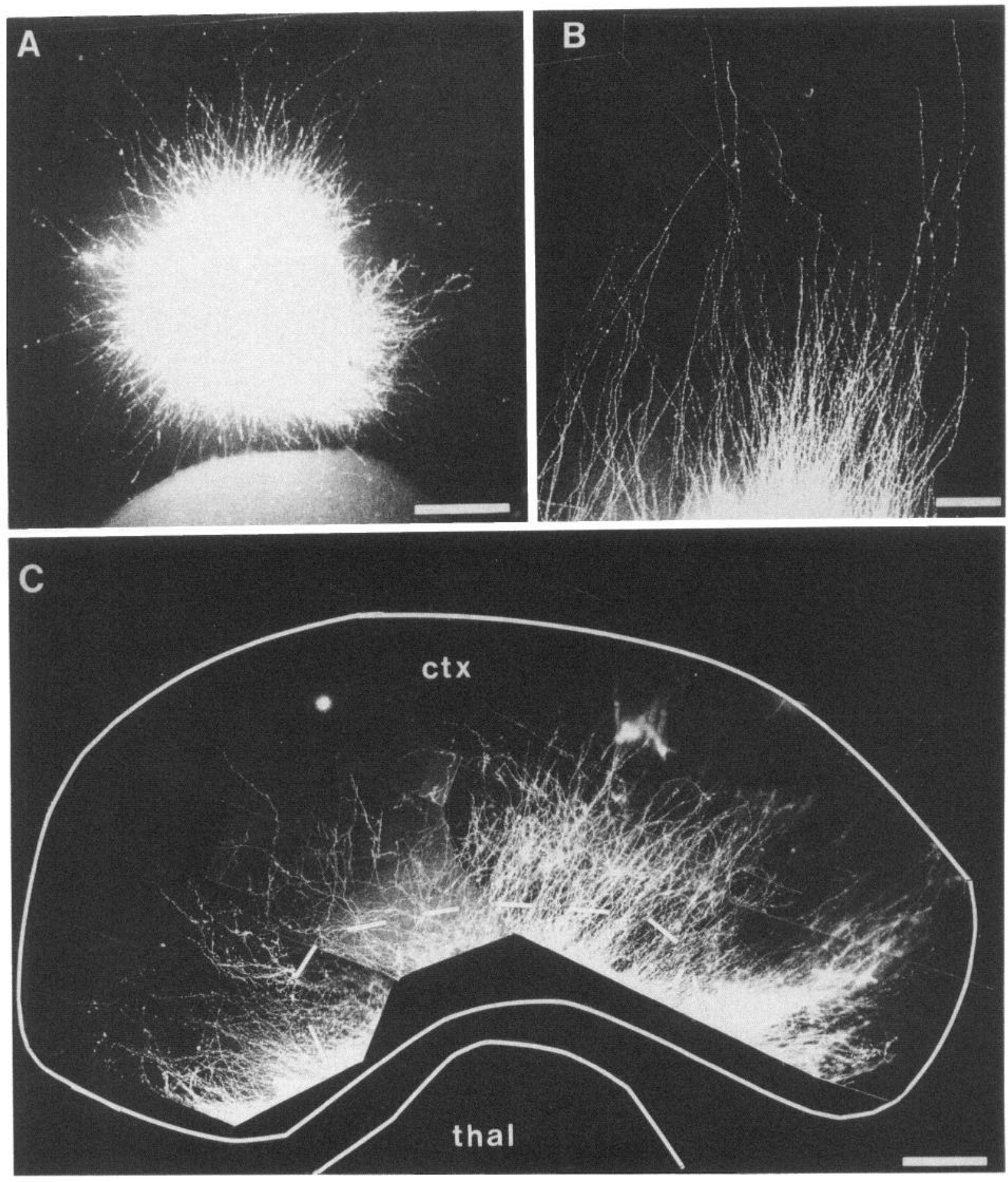

Figure 5. Fluorescent micrographs to illustrate fiber outgrowth from thalamic explants labeled with DiI. $A$, Thalamic fibers leave the explant radially in a rather straight course. Note that fiber trajectories are not influenced by the cocultured cortex, which is partially visible in the lower part of the micrograph. $B$, High-power view of $A$. $C$, Thalamic fibers growing into a cocultured cortical slice. Note that the fibers terminate in the middle of the cortical slice. ctx, Outline of the cortical culture. The broken line indicates the border between gray and white matter as determined after the culture was stained with cresyl violet. thal, Outline of the thalamic culture facing the white matter side of the cortex. Scale bars: $A$ and $C, 500 \mu \mathrm{m} ; B, 25 \mu \mathrm{m}$.

other hand, thalamic fibers that had grown into the cortex could still be detected after 4 weeks in vitro, the longest culture period used in the present study. Fiber outgrowth also depended strongly on the age of the animal from which the explant was taken. We labeled thalamic axons in 338 cocultures with DiI and observed outgrowing fibers in about $80 \%$ of the thalamic slices taken from animals at the day of birth, in about $60 \%$ of the explants taken from P1 or P2 animals and in less than $30 \%$ of the explants from P3 animals (Fig. 6). Only rarely could we detect fiber outgrowth in thalamic cultures prepared from animals after P3.

Once thalamofugal fibers arrived at the cocultured cortical 


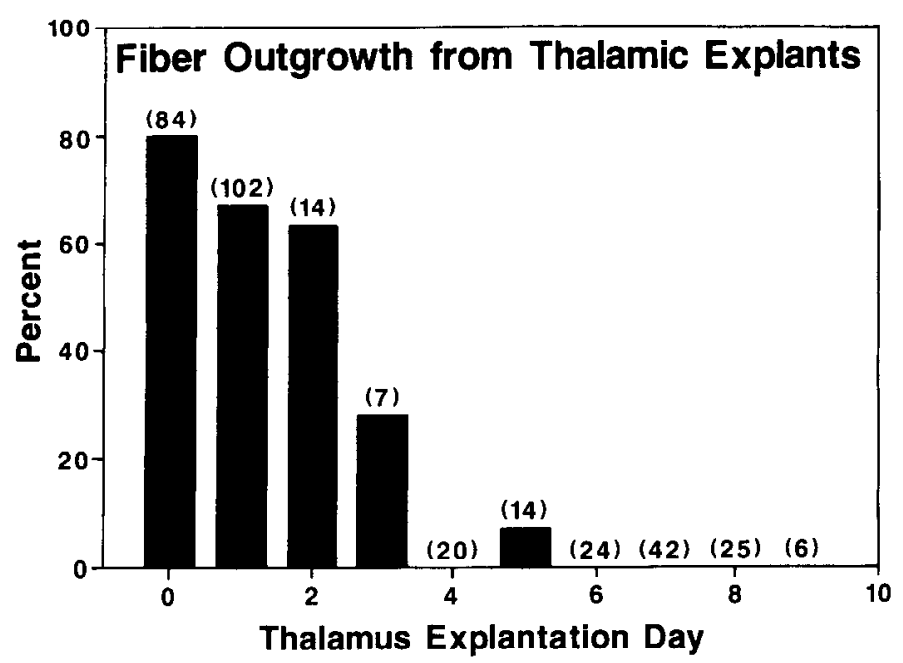

Figure 6. Percentage of thalamic explants where more than five axons were found to grow out. The number of explants examined is indicated above each bar. Almost no fiber outgrowth was observed after P3; note that this time window corresponds to the growth of thalamic axons in vitro.

target tissue after 4-7 DIV, many fibers grew into the cortical slice. However, some fibers did not enter the cortex, but instead grew around the cortical culture. Thalamic fibers were able to invade the cortex from the white matter side (Fig. $5 \mathrm{C}$ ), as well as from the pial side when the thalamus was placed next to the pial surface of the cortical culture (Fig. $7 \mathrm{C}$ ). In both cases, thalamic axons stopped in the middle of the cortical culture and formed terminal arbors that could be quite elaborate (Fig. 8). For a quantitative analysis, we measured the distribution of the endings of all thalamic branches at different relative depths in the cortical explant in four cocultures. A representative example is illustrated in Figurc $7 F$, and Figure $7, D$ and $E$, shows for comparison the distribution of cells labeled with BrdU at E17. We found that $52 \pm 8 \%$ of the thalamic fibers terminate in bins $6-8$, the same position where the majority of BrdU-labeled cells are located. We never observed that thalamic axons grew beyond the cortical explant or that the fibers accumulated at the pial or white matter side of the cultures. When we allowed the cocultures to survive for 1-3 weeks, we often observed thalamic fibers outside the cortical explant in the plasma clot that were much longer than the axons that terminated in the middle of the cortical slices. On the other hand, depending on the distance between the explants, after a short survival time (2-6 DIV), thalamic axons just entered the cortex or did not reach the cortical slice at all. In either case, these thalamic fibers had very few if any branches, in contrast to the thalamic axons that terminated in the middle of the cortical explants.

Fibers emanating from the thalamic cxplants taken from $\mathrm{PO}$ P2 animals invaded cortical slice cultures prepared from $\mathrm{P} 0-$ P9 animals and terminated in the middle of the cortical explants in $44 \%$ of the cocultures examined. The success rate was about the same, irrespective of whether the thalamus was facing the white matter ( 87 cocultures) or the pial side ( 63 cocultures) of the cortical slice cultures. Thalamic explants failed to innervate the cocultured cortex because fibers were only emanating from the thalamus at sides pointing away from the cocultured cortex, or because the cultures were fixed before the thalamic fibers could grow long enough to reach the cortex, or else because thalamic fibers grew around the cortical explants.

\section{Thalamocortical connections in vitro: electrophysiological recordings}

To examine whether ingrowing thalamic axons established functional synapses in the cortical explants, we performed intra- and extracellular recordings in 79 cocultures. In these experiments,
Figure 7. Comparison of the distribution of cortical cells labeled with BrdU at E17, when most cells of layer 4 are born, and the termination of thalamic axons within cortical slice cultures. Drawings of BrdU-labeled cells in a $\mathrm{P} 13$ animal $(A)$ and in a slice culture prepared from a littermate at the day of birth and kept in vitro for $15 \mathrm{~d}(B)$. $C$, Camera lucida drawings of thalamocortical projections in vitro that were anterogradely stained with DiI from the thalamic explant placed close to the pial surface of the cortical slice. $D-F$, Histograms of the position at different relative cortical depths of the BrdU-labeled cells in vivo $(D)$ and in vitro $(E)$, and of the endings of thalamic fibers $(F)$. In these histograms, 0 corresponds to the white matter; 100 , to the pial side of the cortex. The borders between gray and white matter were determined after counterstaining with cresyl violet; they are indicated in $A-C$ by the broken lines. For more details about the layering in cortical slice cultures see Götz and Bolz (1992). Scale bars, $500 \mu \mathrm{m}$.
A

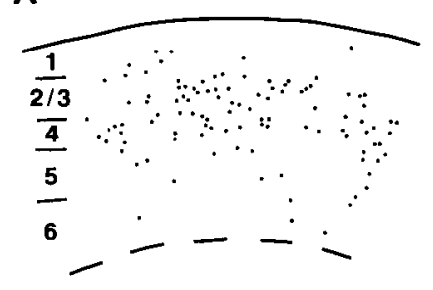

(E17) P13

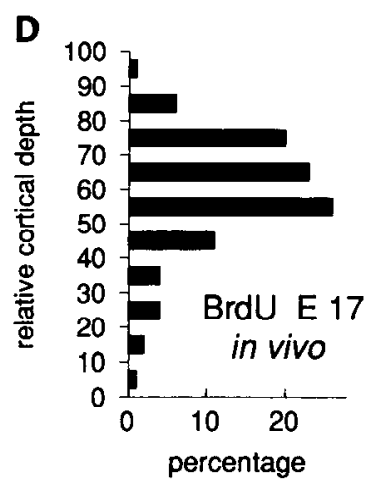

B

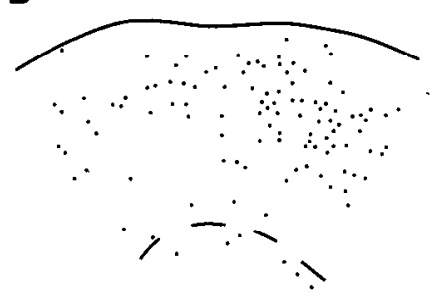

(E17) PO 15DIV

C

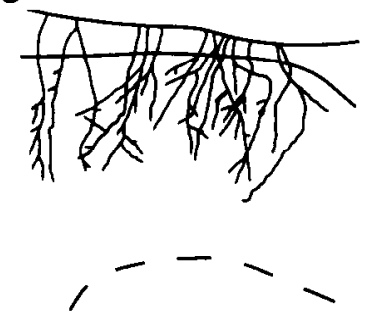

P1 9DIV
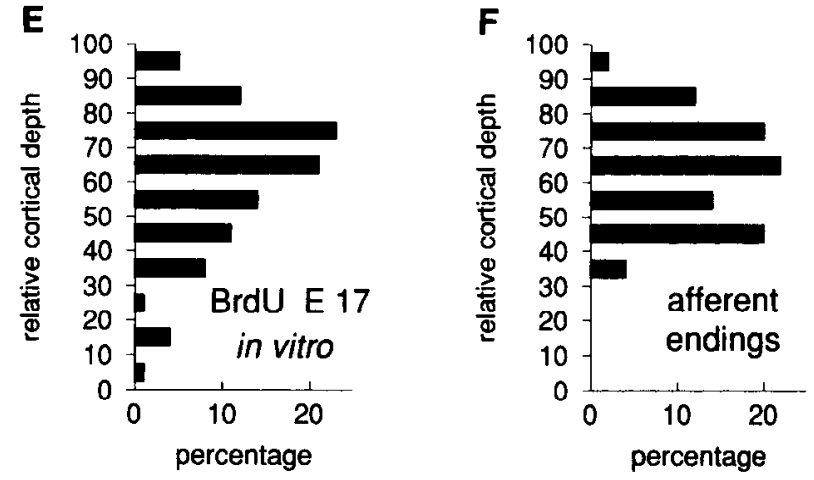

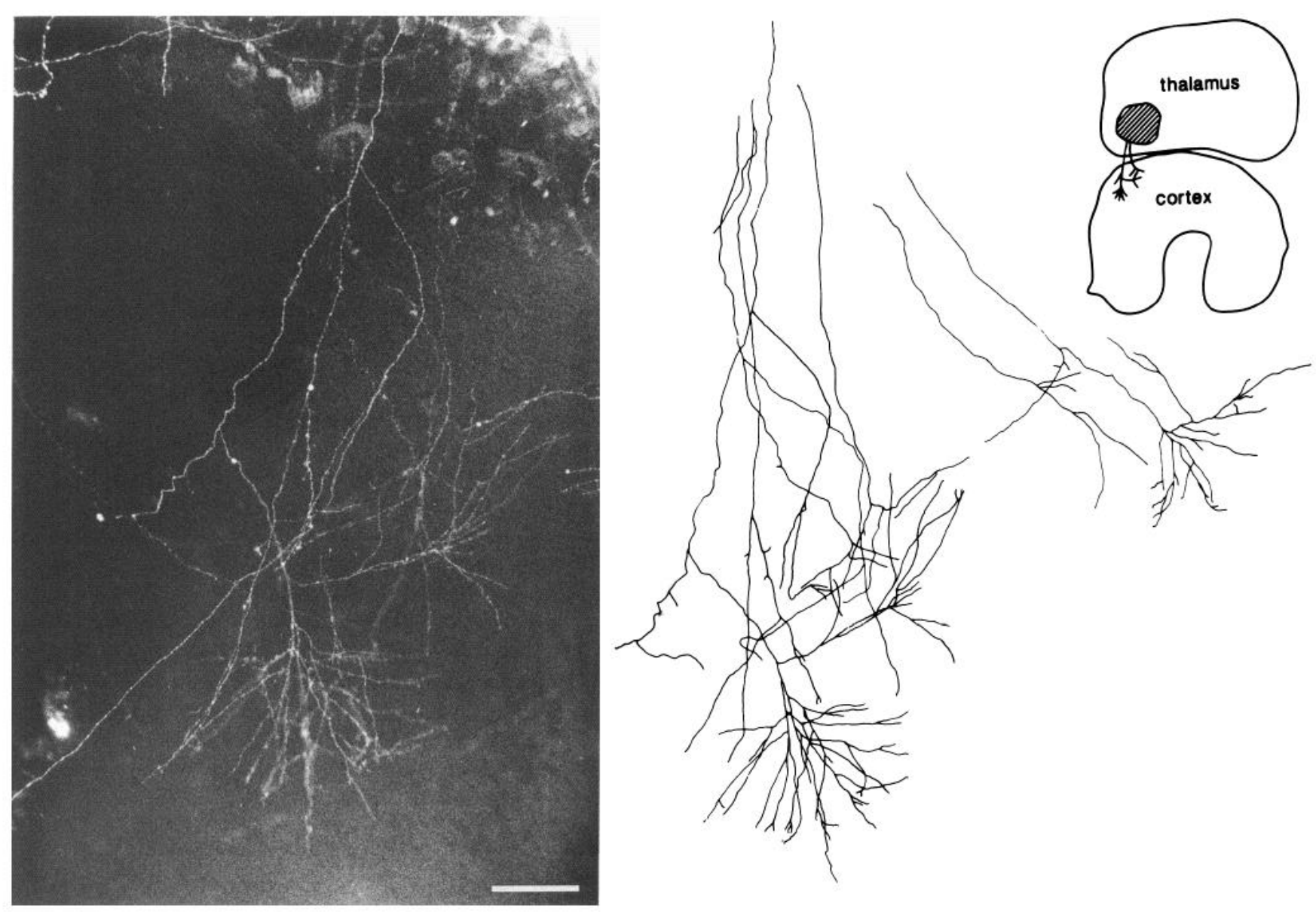

Figure 8. Fluorescent micrograph (left) and camera lucida drawing (right) of axonal arbors of thalamic projection neurons in vitro in a cocultured slice from the visual cortex. The inset (upper right) illustrates that the thalamus was placed toward the pial side of the cortical slice. The shaded area in the thalamus represents a DiI crystal that was inserted to label efferent fibers. Scale bars, $100 \mu \mathrm{m}$.

we placed a recording electrode in the cortical slice and a stimulating electrode at various locations in the cocultured thalamic slice. In 52 out of 133 intracellularly recorded cortical neurons, we were able to elicit a response after electrical stimulation in the thalamus. An example of such an experiment is shown in Figure 9. Stimulation with low intensity evoked an EPSP in the cocultured cortical slice with $17 \mathrm{msec}$ latency, $5 \mathrm{mV}$ amplitude, and about $100 \mathrm{msec}$ duration. When the stimulus intensity was augmented, the EPSP amplitude increased and additional responses occurred that were polysynaptic, since they had longer latencies and were less phase locked to the stimulus than the initial EPSP. When the stimulus intensity was increased further, the number of polysynaptic EPSPs increased, their latencies shortened, and they finally merged with the initial EPSP. Consequently a large, long-lasting EPSP developed that at the highest stimulus intensities used, reached the firing threshold of the cell (upper two traces in Fig. 9).

In Figure 10 are shown different types of synaptic responses from cortical neurons after electrical stimulation in the thalamic explant. In some cells, thalamic stimulation elicited only a fast EPSP with short duration (Fig. 10A), while in others the fast EPSP was followed by a slow, long-lasting component that often reached the cells' firing threshold (Fig. 10B). The latency distribution of the synaptic responses is shown in Figure 11 $A$. In 11 out of 52 cells, only a slow EPSP (latency $>20 \mathrm{msec}$ ) was elicited, and an example of such a response is shown in Figure
$10 \mathrm{C}$. To measure the conduction velocity of thalamic axons in vitro, we placed two extracellular recording electrodes at a defined distance in the cortical explants and stimulated the cocultured thalamus. We found that the conduction velocity was between 0.2 and $0.4 \mathrm{~m} / \mathrm{sec}$. These slow conduction velocities are comparable to the values measured in acute slice preparations from young animals (Komatsu et al., 1981). Thus, EPSPs with latencies shorter than $20 \mathrm{msec}$ are in the range of monosynaptic responses, while EPSPs with latencies longer than 20 msec probably represent polysynaptic responses. Because in a given coculture we could record from only a few cells intracellularly, and because the distance between the thalamic and cortical explants varied considerably between different cocultures, it was not possible to distinguish reliably in our total sample between mono- and polysynaptic responses on the basis of the response latency. To address this weakness of single-unit recording, we used optical recording techniques that will be described in the next section.

To demonstrate the synaptic nature of the electrically evoked responses, in seven cocultures we exchanged the normal bathing medium with a $\mathrm{Ca}^{2+}$-free medium. This treatment completely abolished the responses in all cells tested (Fig. 12). In five cases, the responses recovered when the $\mathrm{Ca}^{2+}$-free medium was replaced by normal medium, but two cells were lost before the recovery was complete. These experiments clearly indicate that responses of cortical neurons after thalamic stimulation are me- 
Figure 9. Intracellular recordings from a cortical neuron in vitro after electrical stimulation in the cocultured thalamus. The arrows mark stimulus onset; three to four swecps arc supcrimposed at cach stimulus intensity (indicated on the right). Note that at the highest stimulus intensities used (the two uppermost traces) the response was suprathreshold and a spike was elicited (spikes are truncated in the figure). The inset (left) shows the position of the stimulating electrode (stim) in the thalamic slice (thal) and the recording electrode ( $\mathrm{rec}$ ) in the cocultured cortical slice $(c t x)$. The broken line indicates the border between gray and white matter.
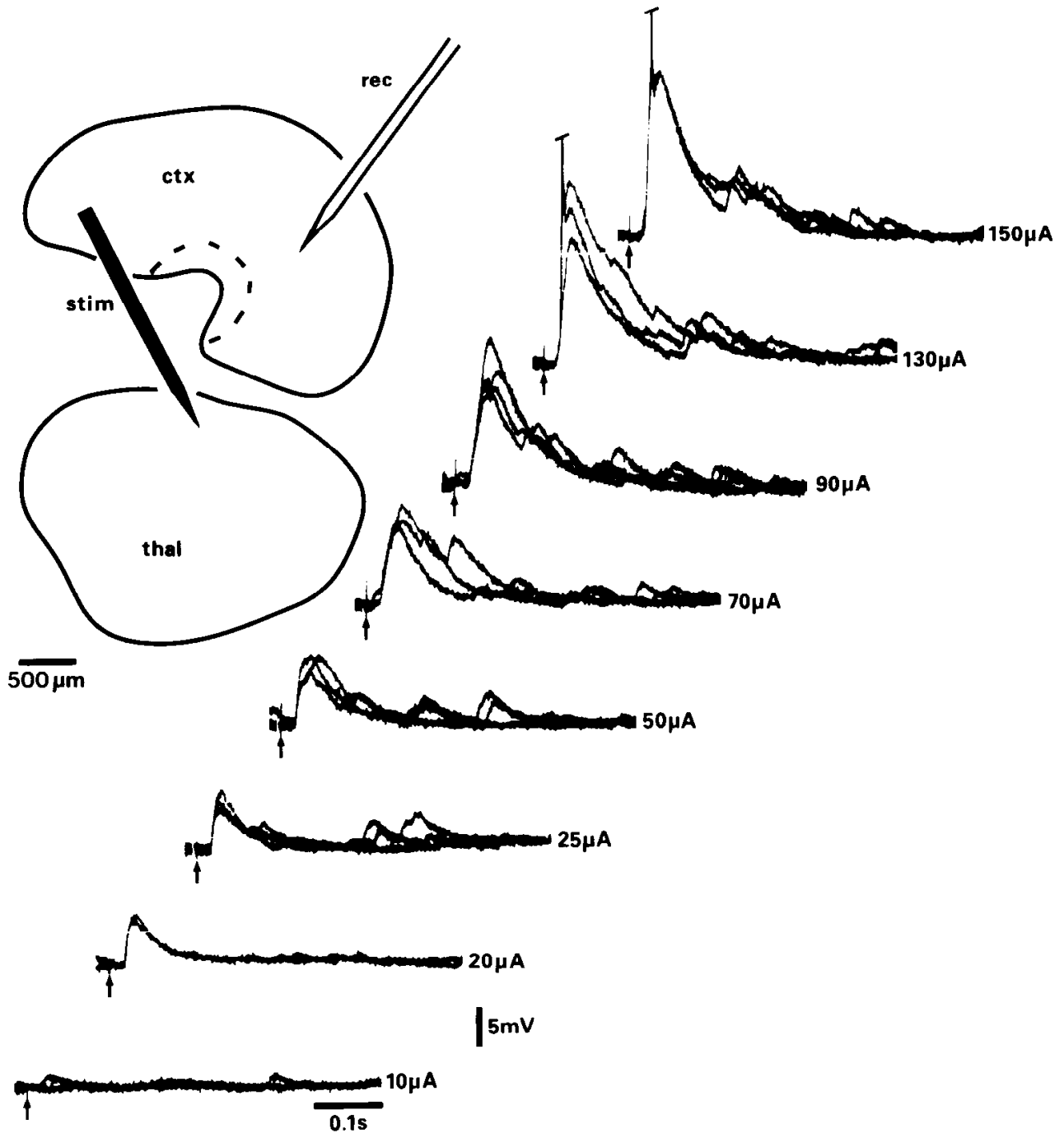

diated by synaptic transmission. However, they do not prove that the responses are due to thalamocortical projection neurons. As was shown previously, corticothalamic cells innervate the cocultured thalamic slices (Yamamoto et al., 1989; Bolz et al., 1990); thus, these cells might be antidromically activated by electrical stimulation in the thalamus and in turn excite other cortical neurons via intrinsic connections.

To rule out the possibility that responses of cortical neurons are due to antidromic activation of corticothalamic neurons, we stimulated thalamic cells pharmacologically in eight cocultures. For this we used iontophoretical application of glutamate, the major excitatory neurotransmitter in the thalamus (Curtis and Davis, 1962; Kemp and Sillito, 1982; Salt, 1987). Since glutamate exerts its postsynaptic excitatory effects on somata and dendrites but not on axons (Hess and Murata, 1974), and since the spread of iontophorized drugs is very restricted (Herz et al., 1969; Bolz et al., 1984), this technique allowed focal stimulation of the thalamus without affecting corticothalamic projection neurons. An example of such an experiment is shown in Figure 13. When the glutamate pipette was placed in the thalamus at the position labeled "glu 1," iontophoretic ejection of glutamate evoked a barrage of EPSPs in the intracellularly recorded cortical neuron. However, when the pipette was moved by $500 \mu \mathrm{m}$ to the position labeled "glu 2," even very high ejection currents for glutamate could not evoke a response in the cortical cell. This demonstrates that focal activation of a restricted region in the thalamus generated postsynaptic responses in the cocultured cortex.

\section{Thalamocortical connections in vitro: optical recordings}

In the preceding section, it was shown that thalamic neurons established functional connections with their cortical target area. Our axonal tracing experiments revealed that thalamofugal fibers arborized predominantly in the middle cortical layers. One would therefore expect that monosynaptic responses should be recorded mainly in the middle of the cortical slices. The distance between the stimulating electrode in the thalamus and the cells recorded in the cortex varied considerably for different recording sites in the cortical slice and even more so for different cocultures. As already mentioned above, this makes it very difficult to distinguish between mono- and polysynaptic latencies, since the latencies of the EPSPs were largely due to the slow conduction velocity. Another difficulty with electrophysiological recordings is that it is nearly impossible to assess whether thalamic stimulation evoked responses at only a few sites in the cortex, or whether thalamocortical connections formed in vitro, in combination with intrinsic cortical connections, are capable of activating a large part of the cocultured cortex. To overcome these 
A
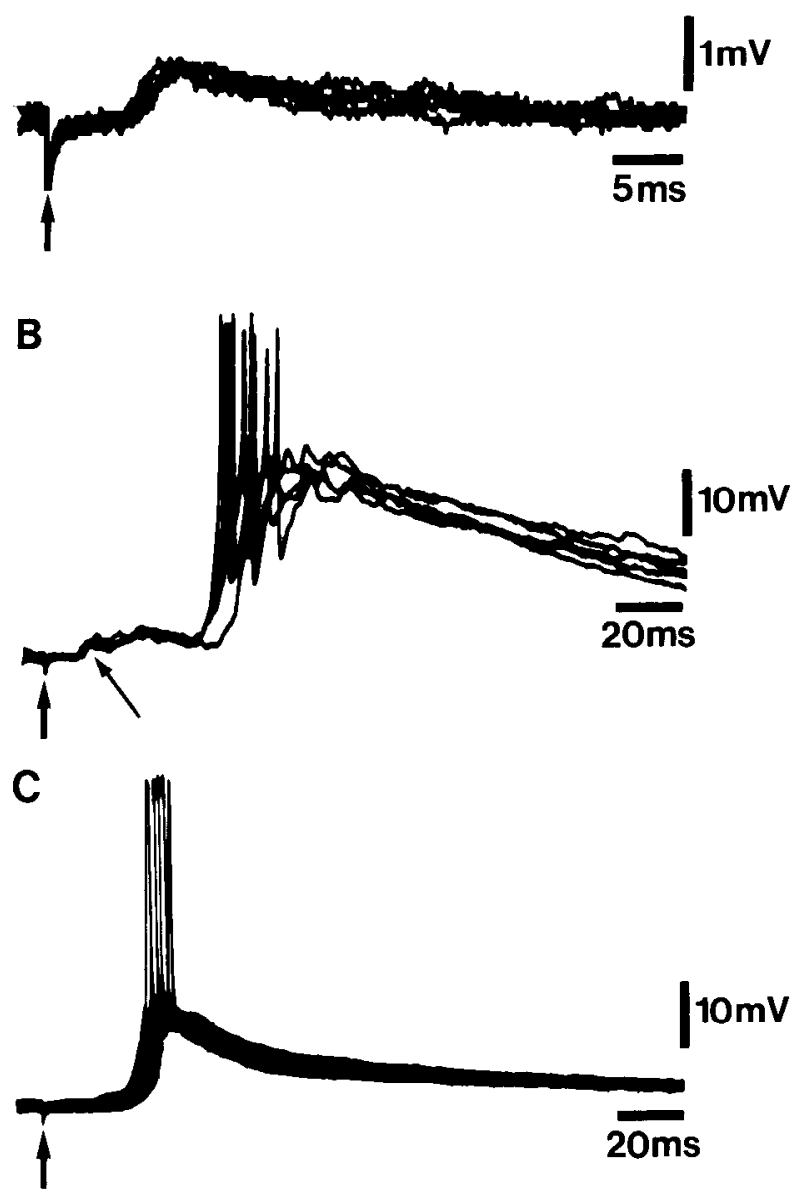

Figure 10. Intracellularly recorded responses of cortical neurons in vitro after electrical stimulation (indicated by short arrows) in the cocultured thalamus. $A$, EPSP with short latency; $B$, EPSP with short latency (long arrow) followed by a large, suprathreshold EPSP with long latency; $C$, suprathreshold EPSP with long latency.

limitations of single-unit recordings, we used voltage-sensitive dyes to monitor neuronal activity with optical techniques. This approach allowed us to detect the electrical activity over the whole cortical slice at high temporal resolution in response to stimulation of the cocultured thalamus.

We performed optical recordings in 22 cocultures, and in 18 cocultures we could record signals in response to electrical stimulation in the thalamus. In the experiment illustrated in Figure 14 , we used a high stimulus intensity that activated about onehalf of the thalamic slice. In response to this strong stimulus, activity was recorded over the whole cortical slice. Figure $14 B$ illustrates the latencies of these responses coded in gray levels, with shorter latencies corresponding to a darker gray. As one might expect, responses with the shortest latencies were recorded in the regions of the thalamus near the stimulating electrode. More importantly, however, in the cortex most responses with short latencies were located in the middle of the slice: $60 \%$ of all recording sites in the middle third of the gray matter had response latencies shorter than $20 \mathrm{msec}$, compared to only $10 \%$ in the upper and $23 \%$ in the lower third of the gray matter. At some recording sites in layer 6 , there were responses with short latencies, these are most clearly visible in the right part of Figure $14 B$. These responses might be due to thalamic fibers inner-
A

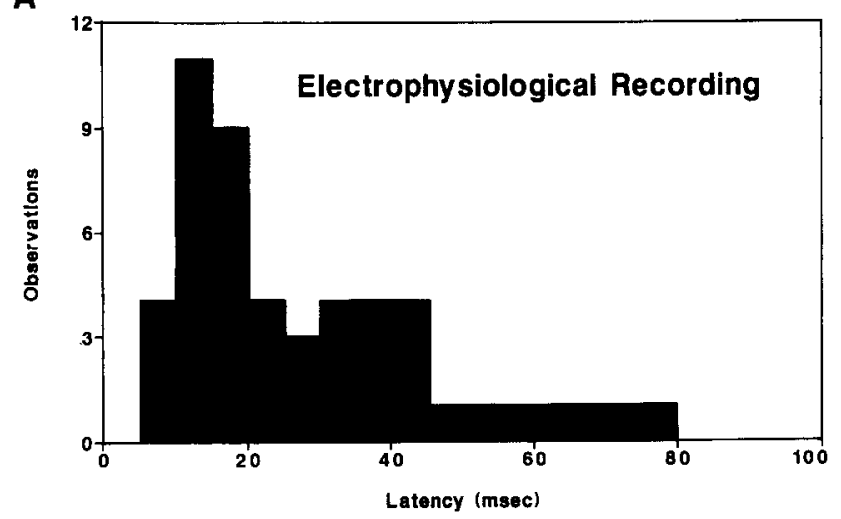

B

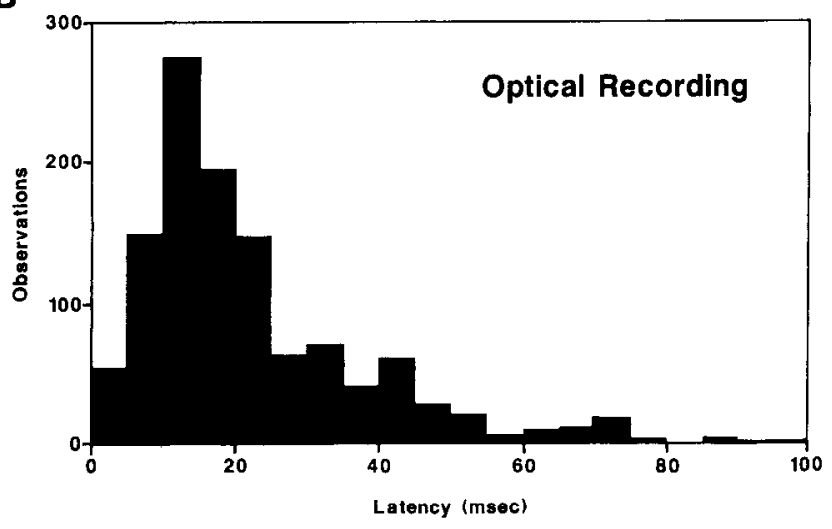

Figure 11. Response latencies of cortical neurons in vitro after stimulation in the cocultured thalamus. $A$, Latencies measured with intracellular recording. $B$, Latencies measured with voltage-sensitive dyes. Note that electrophysiological and optical recording techniques yield comparable results.

vating layer 6 , or they might be due to antidromic activation of corticothalamic neurons, which are known to project to cocultured thalamic explants (Yamamoto et al., 1989; Bolz et al., 1990).

The response latencies measured with optical techniques were very similar to the latencies measured with electrophysiological recordings. Figure 11 depicts the distribution of the latencies measured with these two techniques. Since these data were collected in several different experiments, this figure also indicates that there was a comparable variation of the latencies in the optical and electrophysiological recording experiments.

In the example illustrated in Figure 14 we used a $10 \times$ objective to image the optical signals on the photodiode array. At this low magnification, it was possible to monitor activity over the entire cortical slice by moving the objective to three to six different positions. However, the spatial resolution was relatively poor, since each individual photodiode integrated optical signals over an area of $150 \times 150 \mu \mathrm{m}$. Therefore, in some experiments we used a $63 \times$ objective, where the spatial resolution was $23 \times 23 \mu \mathrm{m}$. A previous study with hippocampal slice cultures demonstrated that at this magnification it is possible to obtain single-cell resolution with optical recording (Bonhoeffer and Staiger, 1988). In the expcriment shown in Figure 15 , we recorded optically with a $63 \times$ objective at nine different positions in the cortical slice. The recording sites were aligned in a column, running from the pial surface down to the white matter side of the cortical culture. In this case, a weak current 


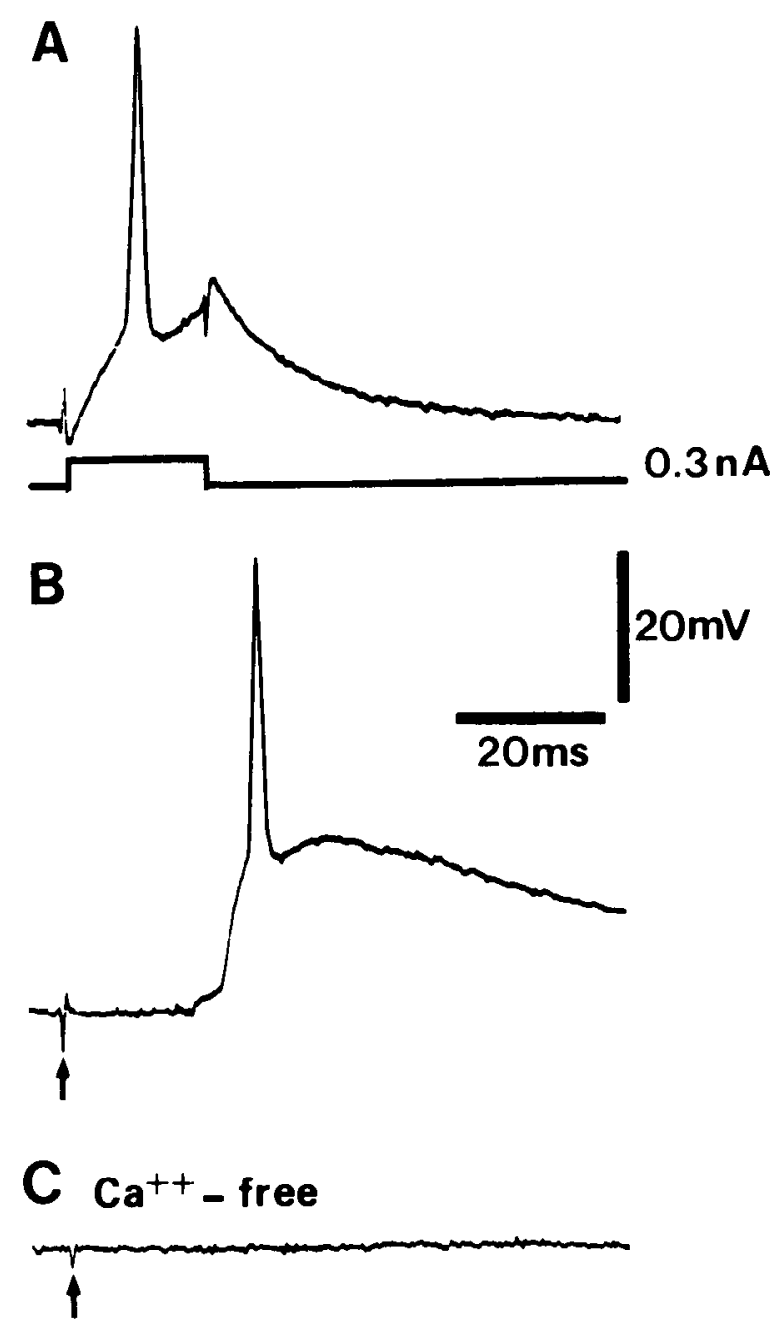

Figure 12. Electrophysiological recordings from a neuron in a cortical slice culture. $A$, Intracellular depolarization to firing threshold with a $0.3 \mathrm{nA}$ current pulse (indicated by the lower trace) elicits a single action potential. $B$, Suprathreshold stimulation in the cocultured thalamus also elicits a single action potential. Note similar shape of the action potential in $A$ and $B . C$, In $\mathrm{Ca}^{2+}$-free medium no response is obtained after electrical stimulation in the thalamus. Short arrows in $B$ and $C$ point to stimulus artifacts.

was applied to the stimulating electrode in the thalamus. As shown on the left side of Figure 15, the shape of the optical signals closely resembled intracellularly recorded electrical responses. Optical responses were recorded only in the middle of the cortical culture, and the responses with the shortest latencies were found in a very narrow band.

\section{Discussion}

In this study, we have examined the formation of thalamocortical projections in a slice culture system. The results show that thalamic neurons make connections with cocultured cortical slices prepared from postnatal animals, where their target cells are located at the appropriate position in layer 4. Connections appeared to be formed by the correct thalamic cell type, since thalamocortical projection neurons in vitro had the morphology of relay neurons, but not of interneurons. The initial outgrowth of thalamic axons in vitro was not influenced by the presence of cortical tissue. However, once thalamic fibers had reached the cortex, they arborized in the appropriate cortical layer and formed functional synaptic contacts. Thalamic axons in vitro made specific connections with their cortical target cells, regardless of whether they entered the cortex from the white matter side or from the pial side. These results suggest that thalamic relay cells have an intrinsic mechanism that allows them to recognize their cortical target cells.

\section{Thalamic development in vitro}

Using a roller culture technique, we obtained viable cultures

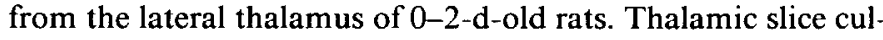
tures prepared from older animals usually degenerated after a few days in vitro. The viability of thalamic cultures was paralleled by the fiber outgrowth from these explants. Fiber outgrowth was observed in thalamic explants taken from 0-2d-old animals, but very rarely in explants taken from older animals. In the rat, during normal development, geniculocortical axons have already arrived beneath the cortical plate by E18, but they do not grow into the cortex. Rather, they wait in the subplate zone until, after a second growth spurt, they enter the cortex and reach their target cells in layer 4 by P1-P3 (Lund and Mustari, 1977). This timing coincides with the capacity of thalamic neurons in vitro to extend neuronal processes and to innervate cocultured cortical tissue.

The two classes of neurons described in rat LGN by Grossman et al. (1973), class A (multipolar cells) and class B (bipolar cells), could be identified in slice cultures, and furthermore they had the characteristic morphology of their in vivo counterparts. In the normal animal, the morphology of both class A and class B cells is very immature during the first 3 postnatal days, the time period during which most thalamic cultures were prepared. Golgi studies showed that cells at this stage of development have short primary dendrites with few higher-order dendrites. In addition, there are often growth cones at the ends and along the stems of dendrites, and fine appendages can be seen arising from the cells' somata (Parnavelas et al., 1977). These immature features were not observed in our slice cultures. After 8 or more days in vitro, thalamic neurons had a rather complex dendritic tree, and we rarely found growth cones on dendrites or appendages on cell bodies. This indicates that the morphological maturation of thalamic neurons continues at least to some extent in vitro. Similar observations have been made previously in slice cultures from the hippocampus, cerebellum, and cortex (Gähwiler, 1981b; Caeser et al., 1989).

\section{Trophic interactions between thalamus and cortex?}

It has been proposed that survival and neurite outgrowth of thalamic neurons depend on the action of factors derived from their cortical target (Haun and Cunningham, 1984; Molnar and Blakemore, 1991). Such trophic influences of target structures on neuronal survival and differentiation during development are well established in the PNS (Levi-Montalcini, 1987). However, the evidence for trophic interactions between thalamic neurons and their cortical targets is less compelling. In vivo studies showed that lesions of the visual cortex during early postnatal development cause massive cell death in the LGN (Cunningham et al., 1979; Perry and Cowey, 1979). After transplantation of cortical explants into the cavity created by lesions of the occipital cortex, the survival time of cells in the LGN was prolonged for 1 week, but nevertheless the neurons died (Haun and Cunningham, 1984, 1987; Cunningham et al., 1987). With dissociated thalamic neurons in vitro, it was found that cocultured cortical cells supported the survival of thalamic neu- 

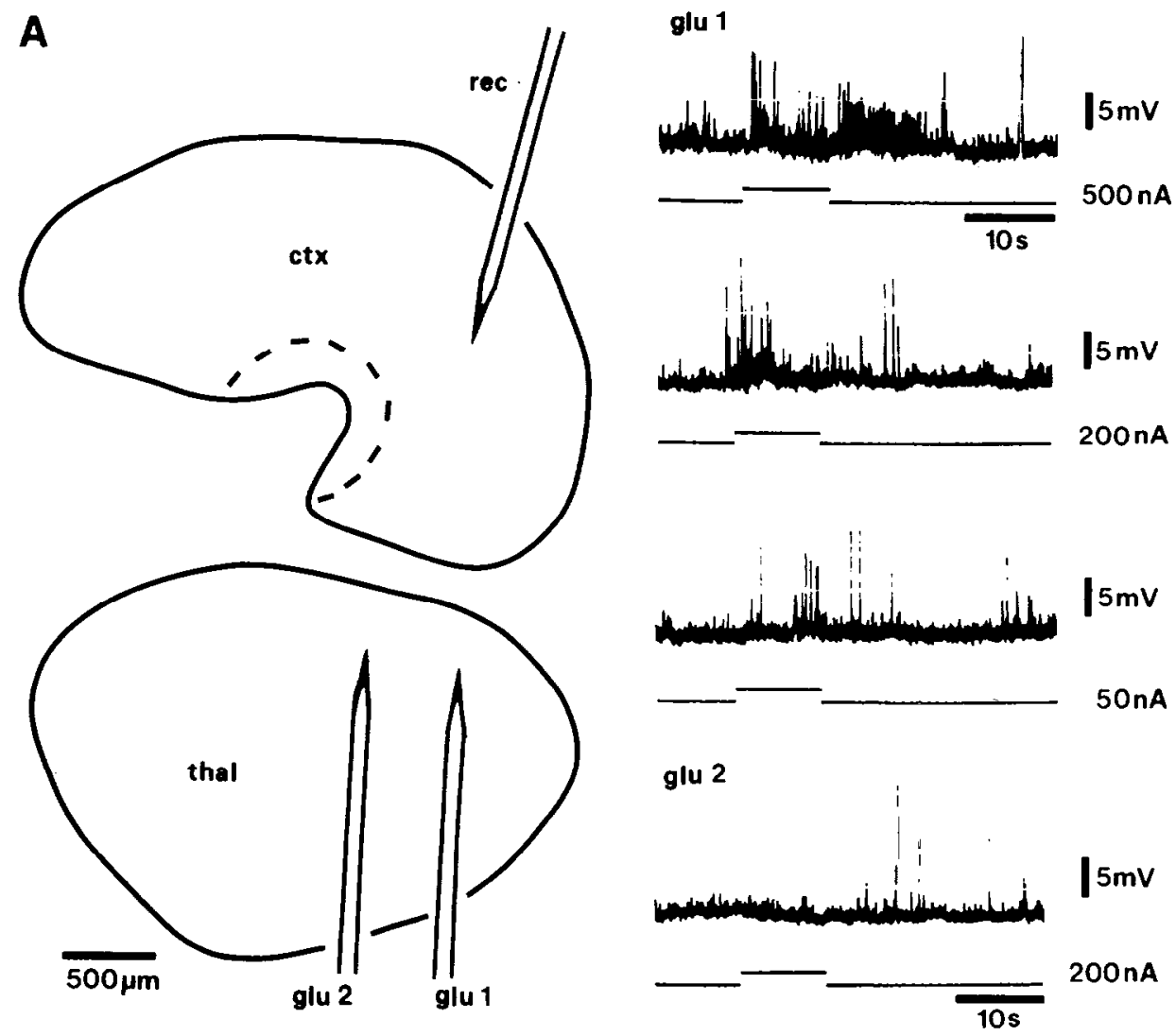

B

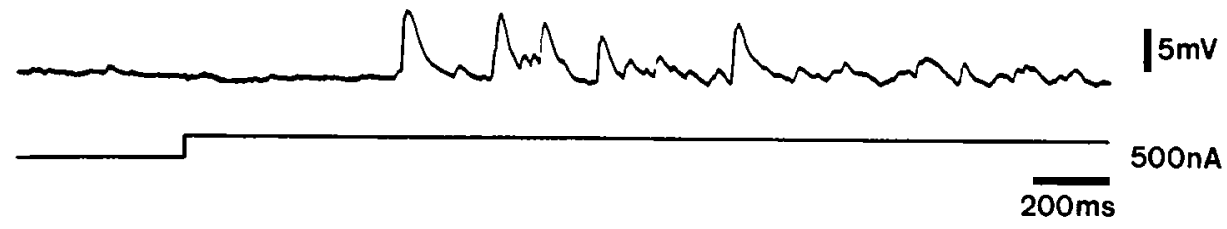

Figure 13. Intracellular recordings from a cortical neuron in vitro after pharmacological stimulation in the cocultured thalamus. Thalamic neurons were excited by iontophoretic application of glutamate. A: Left, The position of the recording electrode in the cortex; the iontophoresis electrode was placed at two different positions in the thalamus labeled glu 1 and glu 2 (same conventions as in Fig. 9). Right, The upper three traces depict responses with the iontophoresis electrode placed at position glu 1 . The duration of the iontophoresis current is indicated below each recording trace, and the strength of the current is shown on the right. No response was obtained with the iontophoresis electrode placed at the position glu 2 (lowest trace). $B$, Expanded time scalc to demonstratc that the responses consist of a barrage of EPSPs. rons, but nontarget cerebellar cells had a much stronger effect on the survival rate of thalamic cells in culture (Hisanaga and Sharp, 1990). Thus, these experiments argue against a specific trophic action of the cortical target on thalamic neurons. Recently, Molnar and Blakemore (1991) reported that thalamic explants on collagen-coated membranes showed very little outgrowth, whereas many thalamic fibers grew into a neighboring cortical explant. This seems to suggest that the cortex can exert a trophic influence on thalamic neurons. However, an alternative interpretation is that these results reflect substrate differences for neurite extension. In contrast to cortical tissue, collagen might be a nonpermissive substrate for growing thalamic axons.

The difficulties encountered in the present study in obtaining viable thalamic slice cultures from early postnatal animals might be due to the absence of target-derived trophic agents. However, another explanation for the limited survival rate of thalamic explants may be found in the structural organization of the thalamus. The thalamus is primarily a relay station; $80 \%$ of its neurons project to the cortex (Sherman and Koch, 1986). More than half of the synaptic contacts in the LGN are made by retinal and cortical input fibers (Wilson et al., 1984), and there is additional input from several different brainstem nuclei (Moore and Bloom, 1979; Kimura et al., 1981; Pasquier and Villar, 1982). Therefore, neurons in thalamic explants are severely de- prived of the inputs they receive and of the contacts they make with other neurons. This is in contrast to cortical explants, since the majority of cortical connections are intrinsic in nature. For example, about $80 \%$ of all synaptic contacts in layer 4 , the main recipient zone of thalamic input, arise from intrinsic cortical connections (White, 1979). Even though there is approximately the same percentage of projection neurons in the cortex as in the thalamus (Gabbott and Somogyi, 1986; Meinecke and Peters, 1987), cortical projection neurons also participate heavily in intrinsic connections. Corticothalamic projection cells, for example, have a strong projection to layer 4 (Katz, 1987), and corticotectal cells have many axon collaterals in layer 6 (Hübener and Bolz, 1988; Hübener et al., 1990). Synaptic connections are therefore much better preserved in cortical than in thalamic explants, and this might be at least one of the reasons why cortical slices survived much better in culture than thalamic slices. In cocultures, thalamic neurons that were able to establish projections to their cortical target cells were observed up to 4 weeks in culture, whereas many fibers that grew out of the thalamic explant but did not reach the cortex degenerated after about 1 week in vitro. Thus, it appears that synaptic contacts are important for the survival of neurons under the described culture conditions. This notion is further supported by a recent study with slice cultures from rat somatosensory cortex (Behan et al, 1991). In rodent somatosensory cortex, there is a unique 


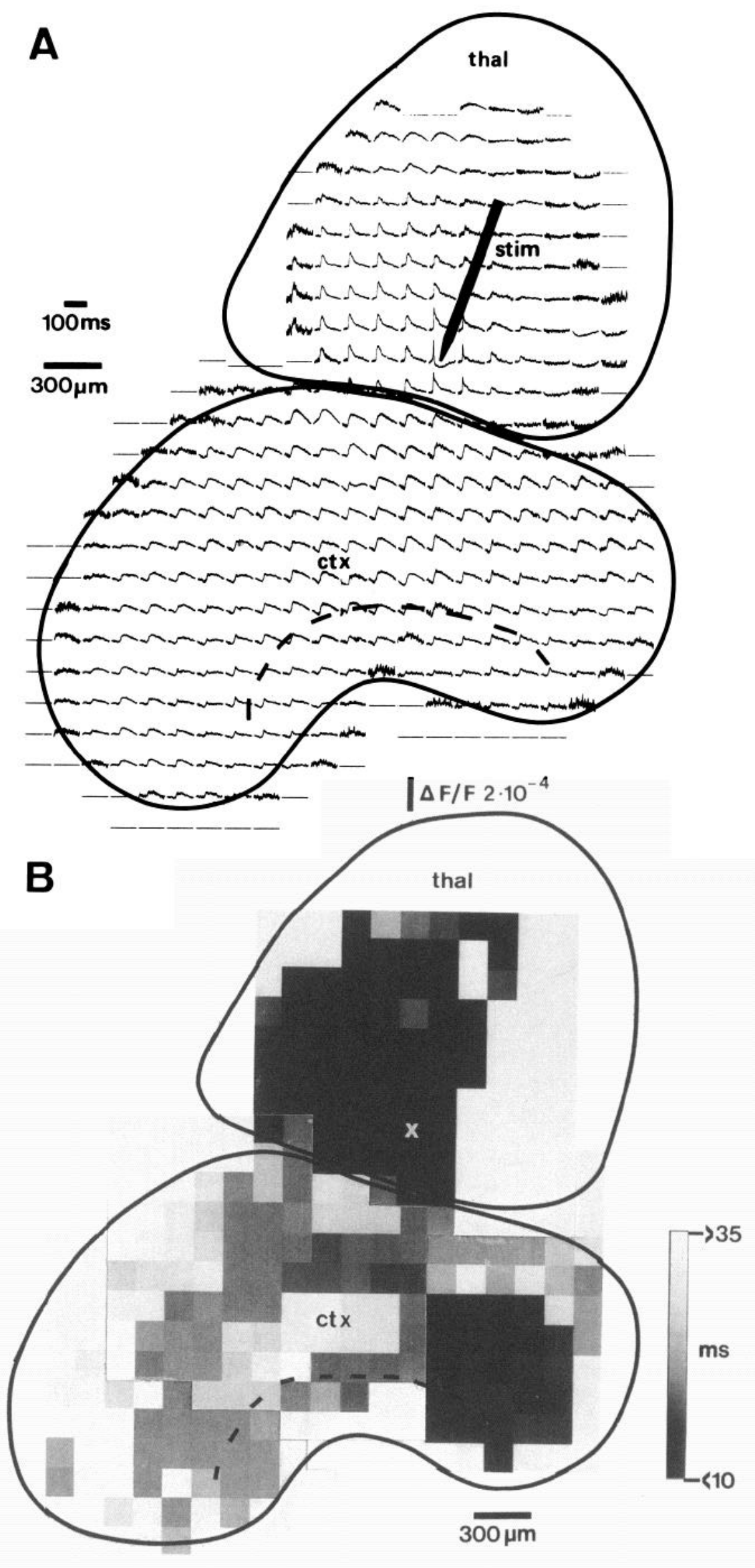

Figure 14. Optical recordings from a cortex-thalamus coculture after electrical stimulation in the thalamus. $A$, Optical signals recorded with a photodiode array after electrical stimulation in the thalamus (same conventions as in Fig. 9). $B$, Latencies of the optical signals coded in gray levels. Note that the shortest latencies are obtained near the stimulating electrode in the thalamus $(x)$ and in the middle of the cortical slice. In this experiment, the thalamus was placed next to the pial side of the cortex. 

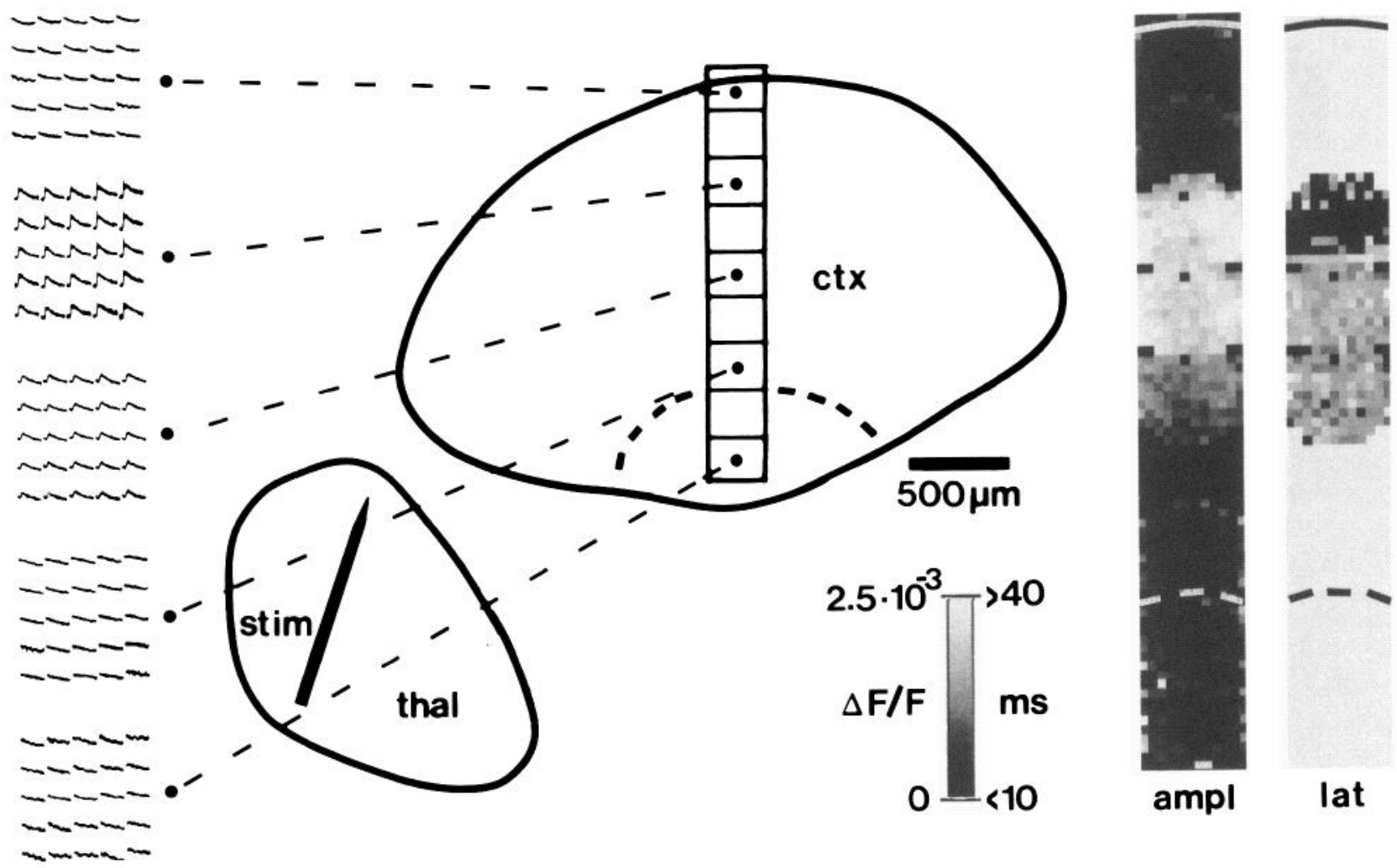

Figure 15. Optical recordings from a cortical slice after application of a weak electrical stimulus in a cocultured thalamic explant. In this experiment, a $63 \times$ objective was used to detect optical signals. The photodiode array was placed at nine different positions as indicated in the outline of the cortical slice culture. Left, Optical signals recorded in the center of the $12 \times 12$ photodiode array. The broken lines point to the corresponding positions in the cortical slice. Right, Amplitude $(\mathrm{ampl})$ and latency $($ lat $)$ coded in gray levels from all recording sites. The pial surface is indicated by the continuous lines (top), and the border between gray and white matter by the broken lines. Note that signals are only recorded in the middle of the cortical slice culture.

organization of groups of neurons in layer 4 into "barrels" (Woolsey and van der Loos, 1970). The barrel architecture was maintained, and most slice cultures survived when the slices were cut sagittally, so that intrinsic interlaminar connections were preserved. However, the barrels disappeared, and many slice cultures degenerated when the slices were cut tangentially, where intrinsic input from other cortical layers was severed. Thus, these results also suggest that intact neuronal circuits are an important factor for the viability of slice cultures.

\section{Pathfinding of thalamocortical axons}

In the present study, we found no influence of the cortical target tissue on the number of axons emerging from thalamic explants, nor was there any indication of a directed growth of thalamic axons toward the cocultured cortex. Axons emerged radially in all directions from thalamic explants, and only axons that happened to grow toward the cortical explant established connections with their target cells. Thus it seems that cortical tissue in vitro has neither a growth-stimulating or trophic, nor an attractant or tropic effect on thalamocortical projection neurons.

Several mechanisms have been proposed that may direct growing central and peripheral axons. For example, extracellular channels have been hypothesized to guide growing axons mechanically toward their target (Singer et al., 1979; Silver and Sidman, 1980). Extracellular matrix molecules are able to promote neurite outgrowth and, when localized in defined tracks, can orient growing axons (for a review, see Jessell, 1988). Studies on the selection of peripheral pathways by axons of developing motor neurons in chick embryos suggested that different populations of motor neurons recognize specific cues within a restricted region of the limb (Lance-Jones and Landmesser, 1981). Such environmental cues or specific preformed pathways are not present in our culture system, and this might be one possible reason why we observed random outgrowth of thalamocortical axons in vitro.

Another mechanism that might guide axons to their targets is the selective fasciculation with other axons (Bray et al., 1980). If axons grow in association with other axons, existing axonal pathways could serve as a scaffold upon which later growing axons extend. The ability of growth cones to recognize axon tracts is well documented in invertebrates. In grasshopper embryos, the axons of identified neurons grow along specific subsets of axon bundles, and ablation studies have demonstrated that these axons may require their preferred axon bundle in order to find their target (Goodman et al., 1984). In the case of the thalamocortical projection, thalamic axons could fasciculate with corticothalamic axons and track along this pathway toward their target. In our in vitro system, we often found that axons of cortical neurons grew into thalamic explants (Bolz et al., 1990); however, we never observed thalamic fibers fasciculating with or growing along these corticothalamic axons. Moreover, in some cocultures thalamic fibers invaded the cortex, but no corticofugal axons were detected. Thus, it appears unlikely that the thalamocortical projection we observed was guided by, or dependent on, the presence of corticothalamic axons.

Although in the present experiments we found no evidence 
for chemoattractant guidance cucs, it is nevertheless conceivable that thalamic axons in vivo might be directed by such a mechanism. Perhaps we failed to detect tropic effects for methodological reasons. If axons are guided by gradients of diffusible molecules emanating from their targets, then these gradients might be diluted or washed out under culture conditions. However, the explants were cultured in plasma clots, and such semisolid matrices appear to stabilize gradients of diffusible molecules secreted from the target (Ebendal and Jacobson, 1977). It seems unlikely that we missed chemoattractant factors for thalamic cells, since the existence of such factors could be demonstrated in the same culture system for cortical projection cells (Bolz et al., 1990). However, during development guidance factors might be secreted by intermediate guideposts rather than by the target itself. Chemotropic guidance by an intermediate target has been demonstrated for spinal commissural axons (Tessier-Lavigne et al., 1988; Placzck et al., 1990). The CNS could employ a similar strategy for the formation of thalamocortical projections, for example by using the internal capsule, the gateway between thalamus and cortex, as a guidepost. This could be tested by coculturing thalamic explants with different subcortical structures that might possibly guide thalamocortical axons.

\section{Target recognition of thalamocortical axons}

A major finding of this study is that thalamic neurons in vitro are able to recognize their correct target layer. Thalamic axons established functional connections in the appropriate layer of cocultured cortical slices, irrespective of whether they invaded the cortex from the white matter or from the pial side of the cortex. Thalamic fibers that did not grow toward a cocultured cortex continued to grow over several millimeters in the plasma clot, and these fibers were usually much longer than those that terminated in the cortical explants. Thus, the termination of thalamic fibers in the middle of cocultured cortical slices is not due to a limited growth capacity of thalamic axons in vitro. Moreover, thalamic axons that grew into the cortex arborized in their target layer. This is in contrast to thalamic axons in the plasma clot, which bifurcated only very rarely. Thus, the cortical target cells in layer 4 appear to provide a signal that causes thalamocortical axons to stop with axon elongation, and to start to branch and to make synaptic contacts.

There have been several speculations about possible interactions between early-arriving thalamocortical axons and their cortical environment. For example, it has been hypothesized that interactions between thalamic afferents and migrating layer 4 neurons might be important in determining that these neurons will later receive the input from the thalamus (McConnell, 1988). In addition, there have been several suggestions that subplate cells mediate processes that might be relevant for the formation of thalamocortical projections (Rakic, 1977; Kostovic and Rakic, 1980, 1990; Shatz and Luskin, 1986; Chun and Shatz, 1988; McConnell et al., 1989; Friauf et al., 1990; Ghosh et al., 1990). Our in vitro experiments with ectopically positioned thalamic explants suggest that thalamic axons have to bypass neither migrating neurons nor subplate cells in order to be able to contact their cortical target cells. Rather, it seems that thalamic neurons have an intrinsic mechanism that allows them to recognize their appropriate cortical cells.

In the present study, thalamic explants were prepared from young postnatal animals, at a time when most geniculocortical axons in vivo accumulate in the subplate zone and migrating layer 4 cells already have passed the waiting afferents. Thus, some critical interactions between thalamic afferents and the cortical environment might have already taken place before slice culture preparation. However, we have preliminary observations with thalamic explants cultured at E16 indicating that neurons from these explants also innervate postnatal cortical slices in the appropriate layer (Götz et al., 1991). These thalamic afferents could not interact with cortical cells, since at this early stage of development in vivo, only very few thalamic axons have arrived in the vicinity of the cortical plate (Lund and Mustari, 1977; Catalano et al., 1991). Thus, it appears that thalamocortical axons are already able to recognize their target cells in layer 4 very early in development. However, this certainly does not exclude the possibility that interactions between thalamic afferents and cortical neurons play a significant role in the formation of thalamocortical projections. For example, in our experiments with embryonic tissue, we found that thalamic afferents are not able to innervate cortical slices where layer 4 cells are not yet present. This suggests that the cortex regulates the innervation of thalamic afferents and directs thalamic fibers at the appropriate time toward their cortical target cells. In further studies, slice cultures should provide a valuable tool to examine more closely the interactions between thalamic afferents and different types of cortical neurons during eslablishment of thalamocortical projections.

\section{References}

Angevine JB, Sidman RL (1961) Autoradiographic study of cell migration during histogenesis of cerebral cortex in the mouse. Nature 192:766-768.

Annis CM, Edmond J, Robertson RT (1990) A chemically-defined medium for organotypic slice cultures. J Neurosci Methods 32:6370.

Behan M, Kroker A, Bolz J (1991) Cortical barrel fields in organotypic slice cultures from rat somatosensory cortex. Neurosci Lett 133:191194.

Berry M, Rogers AW (1965) The migration of ncuroblasts in the developing cerebral cortex. J Anat 99:691-709.

Bolz J, Wässle H, Thier P (1984) Pharmacological modulation of on and off ganglion cells in the cat retina. Neuroscience 12:875-885.

Bolz J, Novak N, Götz M, Bonhoeffer T (1990) Formation of targetspecific neuronal projections in organotypic slice cultures from rat visual cortex. Nature 346:359-362.

Bonhoeffer T, Staiger V (1988) Optical recording with single cell resolution from monolayered slice cultures of rat hippocampus. Neurosci Lett 92:259-264.

Bray D, Wood P, Bunge RP (1980) Selective fasciculation of nerve fibers in culture. Exp Cell Res 130:241-250.

Caeser M, Bonhoeffer T, Bolz J (1989) Cellular organization and development of slice cultures from rat visual cortex. Exp Brain Res 77: 234-244.

Catalano SM, Robertson RT, Killackey HP (1991) Early ingrowth of thalamocortical afferents to the neocortex of the prenatal rat. Proc Natl Acad Sci USA 88:2999-3003.

Chun JJM, Shatz CJ (1988) A fibronectin-like molecule is present in the developing cat cerebral cortex and is correlated with subplate neurons. J Cell Biol 106:857-872.

Chun JJM, Shatz CJ (1989a) Interstitial cells of the adult neocortical white matter are the remnant of the early generated subplate neuron population. J Comp Neurol 282:555-569.

Chun JJM, Shatz CJ (1989b) The earliest-generated neurons of the cat cerebral cortex: characterization by MAP2 and neurotransmitter immunohistochemistry during fetal life. J Neurosci 9:1648-1667.

Chun JJM, Nakamura MJ, Shatz CJ (1987) Transient cells of the developing mammalian telencephalon are peptide-immunoreactive neurons. Nature 325:617-620.

Crain SM, Bornstein MB (1964) Bioelectric activity of mouse cerebral cortex during growth and differentiation in tissue culture. Exp Neurol $10: 425-450$.

Cunningham TJ, Huddelston C, Murray M (1979) Modification of 
neuron numbers in the visual system of the rat. J Comp Neurol 184: $423-434$.

Cunningham TJ, Haun F, Chantler PD (1987) Diffusible proteins prolong survival of dorsal lateral geniculate neurons following occipital cortex lesions in newborn rats. Dev Brain Res 37:133-141.

Curtis DR, Davis R (1962) Pharmacological studies upon neurones of the lateral geniculate nucleus of the cat. Br J Pharmacol 18:217246.

Ebendal T, Jacobson CO (1977) Tissue explants affecting extension and orientation of axons in cultured chick embryo ganglia. Exp Cell Res 105:379-387.

Friauf E, McConnell SK, Shatz CJ (1990) Functional synaptic circuits in the subplate during fetal and early postnatal development of cat visual cortex. J Neurosci 10:2601-2613.

Gabbott PLA, Somogyi P (1986) Quantitative distribution of GABAimmunoreactive neurons in the visual cortex (area 17) of the cat. Exp Brain Res 61:323-331.

Gähwiler BH (1981a) Organotypic monolayer cultures of nervous tissue. J Neurosci Methods 4:329-342.

Gähwiler BH (1981b) Morphological differentiation of nerve cells in thin organotypic cultures derived from rat hippocampus and cerebellum. Proc Natl Acad Sci USA 211:287-290.

Ghosh A, Antonini A, McConnell SK, Shatz CJ (1990) Requirement for subplate neurons in the formation of thalamocortical connections. Nature 347:179-181.

Godement P, Vanselow J, Thanos S, Bonhoefler F (1987) A study in developing visual systems with a new method of staining neurones and their processes in fixed tissue. Development 101:697-713.

Goodman CS, Bastiani MJ, Doe CQ, du Lac S, Helfand SL, Kuwada JY, Thomas JB (1984) Cell recognition during development. Science 225:1271-1279.

Götz M, Bolz J (1989) Development of vasoactive intestinal polypeptide (VIP)-containing neurons in organotypic slice cultures from rat visual cortex. Neurosci Lett 107:6-11.

Götz M, Bolz J (1990) Formation of cortical layers in organotypic slice cultures of rat visual cortex. In: Proceedings of the 18 th Göttingen neurobiology conference, Brain-perception-cognition (Elsner $\mathrm{N}$, Roth G, eds), p 282. Stuttgart: Thieme.

Götz M, Bolz J (1992) Formation and preservation of cortical layers in slice cultures. $J$ Neurobiol, in press.

Götz M, Novak N, Bolz J (1991) Development of afferent projections in rat visual cortex in vivo and in vitro. Soc Neurosci Abstr 17:898.

Grinvald A, Hildesheim R, Farber IC, Anglister L (1982) Improved fluorescent probes for the measurement of rapid changes in membrane potential. Biophys J 39:301-308.

Grinvald A, Anglister L, Freeman JA, Hildesheim R, Manker A (1984) Real-time optical imaging of naturally evoked electrical activity in intact frog brain. Nature 308:848-850.

Grossman A, Lieberman AR, Webster KE (1973) A golgi study of the rat dorsal lateral geniculate nucleus. J Comp Neurol 150:441-466.

Haun F, Cunningham TJ (1984) Cortical transplants reveal CNS trophic interactions in situ. Dev Brain Res 15:290-294.

Haun F, Cunningham TJ (1987) Specific neurotrophic interactions between cortical and subcortical visual structures in developing rat: in vivo studies. J Comp Neurol 256:561-569.

Heffner CD, Lumsden AGS, O'Leary DDM (1990) Target control of collateral extension and directional axon growth in the mammalian brain. Science 247:217-220.

Herz A, Zieglgänsberger W, Färber G (1969) Microelectrophoretic studies concerning the spread of glutamic acid and GABA in brain tissue. Exp Brain Res 9:221-235.

Hess R, Murata K (1974) Effects of glutamate and GABA on specific response properties of neurones in the visual cortex. Exp Brain Res 21:285-297.

Hicks SP, D'Amato CJ (1968) Cell migration to the isocortex in the rat. Anat Rec 160:619-634

Hisanaga K, Sharp FR (1990) Marked neurotrophic effects of diffusible substances released from non-target cerebellar cells on thalamic neurons in culture. Dev Brain Res 54:151-160.

Hübener M, Bolz J (1988) Morphology of identified projection neurons in layer 5 of rat visual cortex. Neurosci Lett 94:76-81.

Hübener M, Schwarz C, Bolz J (1990) Morphological types of projection neurons in layer 5 of cat visual cortex. J Comp Neurol 301: 655-674.

Jessell TM (1988) Adhesion molecules and the hierarchy of neural development. Neuron 1:1-13.
Katz LC (1987) Local circuitry of identified projection neurons in cat visual cortex brain slices. $J$ Neurosci 7:1223-1249.

Kemp JA, Sillito AM (1982) The nature of the excitatory transmitter mediating $X$ and $Y$ cell inputs to the cat dorsal lateral geniculate nucleus. J Physiol (Lond) 323:377-391.

Kimura H, McGeer PL, Peng JH, McGeer EG (1981) The central cholinergic system studied by choline acetyltransferase immunohistochemistry in the cat. J Comp Neurol 200:151-201.

Komatsu Y, Toyama K, Maeda J, Sakaguchi H (1981) Long-term potentiation investigated in a slice preparation of striate cortex of young kittens. Neurosci Lett 26:269-274.

Kostovic I, Rakic P (1980) Cytology and time of origin of interstitial neurons in the white matter in infant and adult human and monkey telencephalon. J Neurocytol 9:219-242.

Kostovic I, Rakic P (1990) Developmental history of the transient subplate zone in the visual and somatosensory cortex of the macaque monkey and human brain. J Comp Neurol 297:441-470.

Lance-Jones C, Landmesser L (1981) Pathway selection by chick lumbosacral motoneurons during development. Proc R Soc Lond [Biol] 214:1-18.

Levi-Montalcini R (1987) The nerve growth factor 35 years later. Science 237:1154-1162.

Lund RD, Mustari MJ (1977) Development of the geniculocortical pathway in rats. J Comp Neurol 173:289-306.

Luskin MB, Shatz CJ (1985) Neurogenesis of the cat's primary visual cortex. J Comp Neurol 242:611-631.

McConnell SK (1988) Development and decision-making in the mammalian cerebral cortex. Brain Res 13:1-23.

McConnell SK, Ghosh A, Shatz CJ (1989) Subplate neurons pioneer the first axon pathway from the cerebral cortex. Science 245:978982.

Meinecke DL, Peters A (1987) GABA immunoreactive neurons in rat visual cortex. J Comp Neurol 261:388-404.

Miller MW (1988) Development of projection and local circuit neurons in neocortex. In: Cerebral cortex, Vol 7; Development and maturation of cerebral cortex (Peters A, Jones EG, eds), pp 133-175. New York: Plenum.

Miller MW, Nowakowski RS (1988) Use of bromodeoxyuridine-immunohistochemistry to examine the proliferation, migration and time of origin of cells in the central nervous system. Brain Res 457:44-52.

Molnar Z, Blakemore C (1991) Lack of regional specificity for connections formed between thalamus and cortex in coculture. Nature $351: 475-477$

Moore RY, Bloom FE (1979) Central catecholamine neuron systems: anatomy and physiology of the norepinephrine and epinephrine systems. Annu Rev Neurosci 2:113-168.

Parnavelas JG, Mounty EJ, Bradford R, Lieberman AR (1977) The postnatal development of ncurons in the dorsal lateral geniculate nucleus of the rat: a Golgi study. J Comp Neurol 171:481-500.

Pasquier DA, Villar MJ (1982) Specific serotonergic projections to the lateral geniculate body from the lateral cell groups of the dorsal raphe nucleus. Brain Res 249:142-146.

Perry VH, Cowey A (1979) Changes in the retino-fugal pathways following cortical and tectal lesions in neonatal and adult rats. Exp Brain Res 35:97-108.

Placzek M, Tessier-Lavigne M, Yamada T, Jessell T, Dodd J (1990) Mesodermal control of neural cell identity: foor plate induction by the notochord. Science 250:985-988.

Rakic P (1974) Neurons in rhesus monkey visual cortex: systematic relation between time of origin and eventual disposition. Science 183: 425-427.

Rakic P (1977) Prenatal development of the visual system in rhesus monkey. Philos Trans R Soc Lond [Biol] 278:245-260.

Romijn BM, de Jong BM, Ruijter JM (1988) A procedure for culturing rat neocortex explants in a serum-free nutrient medium. J Neurosci Methods 23:75-83.

Salt TE (1987) Excitatory amino acid receptors and synaptic transmission in the rat ventrobasal thalamus. J Physiol (Lond) 391:499510.

Seil FJ, Kelly JM, Leiman AL (1974) Anatomical organization of cerebral neocortex in tissue culture. Exp Neurol 45:435-450.

Shatz CJ, Luskin MB (1986) The relationship between the geniculocortical afferents and their cortical target cells during development of the cat's primary visual cortex. J Neurosci 6:3655-3668.

Sherman SM, Koch C (1986) The control of retinogeniculate trans- 
mission in the mammalian lateral geniculate nucleus. Exp Brain Res 63:1-20.

Silver J, Sidman RL (1980) A mechanism for the guidance and topographic patterning of retinal ganglion axons. J Comp Neurol 189: 101-111.

Singer M, Nordländer RH, Egar M (1979) Axonal guidance during embryogenesis and regeneration in the spinal cord of the newt: the blueprint hypothesis of neuronal pathway patterning. J Comp Neurol 185:1-22.

Tessier-Lavigne M, Placzek M, Lumsden AGS, Dodd J, Jessell TM (1988) Chemotropic guidance of developing axons in the mammalian central nervous system. Nature 336:775-778.

Wahle P, Meyer G (1989) Early postnatal development of vasoactive intestinal polypeptide- and peptide histidine isoleucine-immunoreactive structures in the cat visual cortcx. J Comp Ncurol 282:215248.

Wahle P, Meyer G, Wu J-Y, Albus K (1987) Morphology and axon terminal pattern of glutamate decarboxylase-immunoreactive cell types in the white matter of the cat occipital cortex during early postnatal development. Dev Brain Res 36:53-61.

Webster MJ, Rowe MH (1984) Morphology of identified relay cells and interneurons in the dorsal lateral geniculate nucleus of the rat. Exp Brain Res 56:468-474.

White EL (1979) Thalamocortical synaptic relations: a review with emphasis on the projections of specific thalamic nuclei to the primary sensory areas of the neocortex. Brain Res Rev 1:275-311.

Wilson JR, Friedlander MJ, Sherman SM (1984) Fine structural morphology of identified X-and Y-cells in the cat's lateral geniculate nucleus. Proc R Soc Lond [Biol] 221:411-436.

Wise SP, Jones EG (1976) The organization and postnatal development of the commissural projection of the rat somatic sensory cortex. J Comp Neurol 168:313-344.

Wise SP, Jones EG (1978) Developmental studies of thalamocortical and commissural connections in the rat somatic sensory cortex. J Comp Neurol 175:187-208.

Wolburg H, Bolz J (1991) Ultrastructural organization of slice cultures from rat visual cortex. J Neurocytol 20:552-563.

Wolfson B, Gutnick MJ, Baldino F Jr (1989) Electrophysiological characteristics of neurons in neocortical explant cultures. Exp Brain Res 76:122-130.

Woolsey TA, Van der Loos H (1970) The structural organization of layer IV in the somatosensory region (SI) of mouse cerebral cortex. The description of a cortical field composed of discrete cytoarchitectonic units. Brain Res 17:205-242.

Yamamoto N, Kurotani T, Toyama K (1989) Neural connections between the lateral geniculate nucleus and visual cortex in vitro. Science 245:192-194. 